\title{
Capabilities of Laser Diagnostics for Combustion and Flowfields
}
R. A. Hutchinson
H. J. Bomelburg

November 1984

Prepared for the U.S. Department of Energy under Contract DE-AC06-76RLO 1830

Pacific Northwest Laboratory Operated for the U.S. Department of Energy by Battelle Memorial Institute 


\title{
DISCLAIMER
}

This report was prepared as an account of work sponsored by an agency of the United States Government. Neither the United States Government nor any agency thereof, nor any of their employees, makes any warranty, express or implied, or assumes any legal liability or responsibility for the accuracy, completeness, or usefulness of any information, apparatus, product, or process disclosed, or represents that its use would not infringe privately owned rights. Reference herein to any specific commercial product, process, or service by trade name, trademark, manufacturer, or otherwise, does not necessarily constitute or imply its endorsement, recommendation, or favoring by the United States Government or any agency thereof. The views and opinions of authors expressed herein do not necessarily state or reflect those of the United States Government or any agency thereof.

\author{
PACIFIC NORTHWEST LABORATORY \\ operated by \\ BATTELLE \\ for the \\ UNITED STATES DEPARTMENT OF ENERGY \\ under Contract DE-AC06-76RLO 1830
}

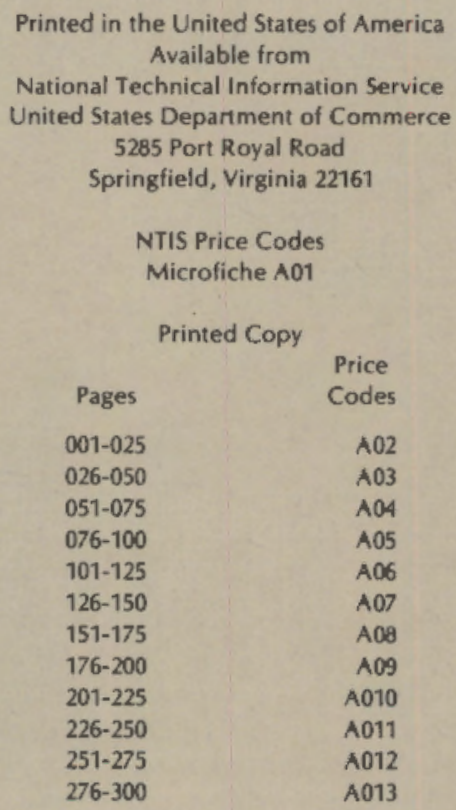


CAPABILITIES OF LASER DIAGNOSTICS FOR COMBUSTION AND FLOWFIELDS

R. A. Hutchinson

H. J. Bomelburg

November 1984

Prepared for Energy Conversion and Utilization Technologies Division Office of Energy Systems Research Conservation and Renewable Energy under Contract DE-AC06-76RLO 1830 for the U.S. Department of Energy

Pacific Northwest Laboratory Richland, Washington 99352 
. 
PREFACE

This study was completed for the Division of Energy Conversion and Utilization Technologies (ECUT) in the Department of Energy. The division's mission is to identify and research long-range technology concepts for more efficient energy use. To meet its goals, the ECUT staff established a planning and systems anaiysis project that was responsible for conducting this study.

This report is one of a series of studies in support of the ECUT research planning effort. This volume examines the capabilities of a powerful new set of measurement techniques for flows in energy conversion systems. This and a companion volume, Applications of Laser Diagnostics in Energy Conservation Research (PNL-5281), also fill a role in technology transfer, providing ideas and information to potential users of laser diagnostic techniques. Other documents in the series contain assessments of energy conservation technology areas, appraisal of research projects for support, and data reference sources. Publications from this project include the following:

Abarcar, R., and D. Johnson. 1984. ECUT Data Reference Series: Lightweight Materials for Ground Transportation. PNL-5192, Pacific Northwest Laboratory, Richland, Washington.

Abarcar, R., and D. Johnson. 1984. ECUT Data Reference Series: High-Temperature Materials for Advanced Heat Engines. PNL-5193, Pacific Northwest Laboratory, Richland, Washington.

Bomelburg, H. J. 1983. Efficiency Evaluation of Oxygen Enrichment in Energy Conversion Processes. PNL-4917, Pacific Northwest Laboratory, Richland, Washington.

Chockie, A., and D. Johnson. 1984. ECUT Data Reference Series: Boilers. PNL-5195, Pacific Northwest Laboratory, Richland, Washington.

Hane, G. 1983. Efficiency Evaluation of the DISC, DHC, and DI Diesel Engines. PNL-4568, Pacific Northwest Laboratory, Richland, Washington.

Hane, G., et al. 1983. A Preliminary Overview of Innovative Industrial Materials Processes. PNL-4505, Pacific Northwest Laboratory, Richland, Washington. 
Hane, G., et al. 1984. A Review of Studies of Research Opportunities in Energy Conservation. PNL-4571, Pacific Northwest Laboratory, Richland, Washington.

Hane, G. and D. Johnson. 1984. ECUT Data Reference Series: Otto Cycle Engines in Transportation. PNL-5191, Pacific Northwest Laboratory, Richland, Washington.

Hopp, W., et al. 1981. An Overview of Energy Conservation Research Opportunities--Executive Summary. PNL-3944, Pacific Northwest Laboratory, Richtand, Washington.

Hopp, W., et a1. 1981. An Overview of Energy Conservation Research Opportunities. PNL-3944, Pacific Northwest Laboratory, Richland, washington.

Hopp, W., et a1. 1982. Identification of Energy Conservation Research Opportunities: A Review and Synthesis of the Literature. PNL-3966, Pacific Northwest Laboratory, Richland, Washington.

U.S. Department of Energy. 1981. The 1981 ECUT Work Element Appraisal. DOE/CE-0024, U.S. Department of Energy, Hashington, D.C.

U.S. Department of Energy. 1983. Energy Conversion and Utilization Technologies Program Report, 1981-1982. U.S. Department of Energy, Washington, D.C.

Vallario, R. W., and D. E. DeBellis, 1984. State of Technology of Direct Contact Heat Exchanging. PNL-5008, Pacific Northwest Laboratory, Richland, Washington.

Vitullo, M., C. Winter and D. R. Johnson. 1984. The Executive Information System. PNL-5190, Pacific Northwest Laboratory, Richland, Washington.

Young, J., and D. Johnson. 1984. ECUT Data Reference Series: Ammonia Synthesis Energy Use and Capital Stock Information. PNL-5194, Pacific Northwest Laboratory, Richland, Washington. 


\section{ACKNOWLEDGMENTS}

The authors extend their appreciation to the many people who contributed to bringing this report to completion. In particular, the authors would like to thank the staff at Sandia-Livermore's Combustion Research Facility, especially Bill Robinson, Mike Dyer, and Taz Bramlette, for their advice throughout this project. Russ Barnes and Tony Boiarski at Battelle Columbus, Dave Crosley at SRI International, Paul Hedman at BYU, and Ron Hanson and Mark Allen at Stanford were also very helpful at various stages of the work. Finally, Sue Liebetrau's editing assistance is greatly appreciated. 

SUMMARY

This report outlines the capabilities and introduces some of the complications of several laser diagnostic techniques that utilize a variety of optical phenomena. Originally developed for aeronautics and analytical chemistry, and more recently for combustion work, these techniques are potentially applicable to a wide, variety of in-situ measurements. The goal of this report is to provide a nonexpert, whether student or researcher, with basic information about the better-established diagnostics. This introduction may lead, after further study, to application of diagnostic techniques to new and important problem areas, particularly those involved in energy conversion.

In the report, considerable emphasis is placed on general concerns in choosing and using a diagnostic and on outlining the limitations of specific diagnostics. These factors, and the decisions and compromises they force, are critical elements in the selection of what to measure and the best tool with which to measure it. They are not generally reported in broad terms in the literature, however. The simple discussion here provides a preliminary, useroriented view of lasers, data acquisition and reduction requirements, generic sources of error, and the difficulties of making good measurements, as well as the required conditions and difficulties of using particular tools.

The tools discussed here can be divided into two optical types: imaging or visualization methods, and light-scattering methods. The former techniques are summarized in Table S.1. They are relatively simple, and although they generaily do not provide quantitative information, they are quite useful as starting points in the examination of an unknown flowfield. They can provide much more information than conventional high-speed photography. They also provide, when combined with holography (a three-dimensional recording technique), the oniy well-established full-field measurement method. Full threedimensional flow information is now regarded as vital to the development of understanding of most complex flows (Goulard 1983).

Light-scattering methods are more complex than imaging methods, and are the basis of most of the diagnostics discussed in this report. These diagnostics provide information ranging from particle size distributions or velocities 


\section{TABLE S.1. Laser Visualizations}

\begin{tabular}{|c|c|c|c|}
\hline Tool & Parameter Measured & $\begin{array}{l}\text { Information } \\
\text { Gained }\end{array}$ & Comments \\
\hline Shadow & $\begin{array}{l}\text { 2nd derivative of } \\
\text { refractive index }\end{array}$ & Density & $\begin{array}{l}\text { Qualitative, in- } \\
\text { expensive, fast }\end{array}$ \\
\hline Schlieren & $\begin{array}{l}\text { 1st derivative of } \\
\text { refractive index }\end{array}$ & Density & $\begin{array}{l}\text { Qualitative, in- } \\
\text { expensive, fast }\end{array}$ \\
\hline Interferometry & Refractive index & $\begin{array}{l}\text { Density (can } \\
\text { also be used } \\
\text { for position) }\end{array}$ & $\begin{array}{l}\text { Provides only } \\
\text { relative } \\
\text { measurement }\end{array}$ \\
\hline
\end{tabular}

to gas temperature, density or species concentration. They are basically nonintrusive and in-situ, and can provide good temporal and three-dimensional spatial resolution. They do, however, require the layman to exert some effort to keep from confusing them.

This confusion can be minimized by separating elastic processes (unshifted in frequency) from inelastic ones, as shown in Table S.2. Elastic processes are focused on particle velocity and density data, although temperature measurements are possible. It should also be noted that the velocity measurements actually utilize a slight inelasticity in the light scattering--the Doppler shift due to particle motion.

The truly inelastic methods can yield species information by comparing the scattered light (which has a characteristic spectrum based on the structure of the species from which it scatters) with known spectra. Special comparisons can also provide sample temperatures. The inelastic methods vary considerably in sensitivity, as the scattering cross-sections in Tabie $S .3$ show. This variance is one of the critical factors in selecting a diagnostic technique. The methods also vary in their ease of use, time resolution, and the environments in which they can be made, although none of these factors is independent.

The complexities of such interrelationships are part of the difficulty in using laser diagnostics. Diagnostics are exceedingly powerful, and a measurement effort must be carefully planned and directed at targets of significance. This report provides a sampling of situations in which diagnostics have been useful despite their limitations. It is increasingly recognized that 
IABLE S.2. Light-Scattering Diagnostic Methods(a)

\begin{tabular}{|c|c|c|c|c|}
\hline Observation & Scatterer & Process & Information & Comments \\
\hline \multirow[t]{4}{*}{$\begin{array}{l}\text { Elastic } \\
\text { (unshifted) } \\
\text { scattering }\end{array}$} & \multirow[t]{2}{*}{ Particles } & \multirow[t]{2}{*}{ Mie } & $\begin{array}{l}\text { Particle } \\
\text { size } \\
\text { distribution }\end{array}$ & $\begin{array}{l}\text { Difficult to } \\
\text { interpret data } \\
\text { if nonided } \\
\text { particles }\end{array}$ \\
\hline & & & Velocity & $\begin{array}{l}\text { Particles must } \\
\text { follow flow }\end{array}$ \\
\hline & \multirow[t]{2}{*}{ Gas } & \multirow[t]{2}{*}{ Rayleigh } & $\begin{array}{l}\text { Total } \\
\text { density }\end{array}$ & $\begin{array}{l}\text { Known compo- } \\
\text { sition, few } \\
\text { particles only }\end{array}$ \\
\hline & & & Temperature & $\begin{array}{l}\text { As above, plus } \\
\text { equilibrium }\end{array}$ \\
\hline \multirow[t]{3}{*}{$\begin{array}{l}\text { Inelastic } \\
\text { (shifted) }\end{array}$} & Gas & Fluorescence & $\begin{array}{l}\text { Temperature, } \\
\text { minor species } \\
\text { densities }\end{array}$ & $\begin{array}{l}\text { Needs clean } \\
\text { environment, } \\
\text { absolute } \\
\text { measurement } \\
\text { difficult }\end{array}$ \\
\hline & $\begin{array}{l}\text { Gas, liquid, } \\
\text { solid surface } \\
\text { or particles }\end{array}$ & Raman & $\begin{array}{l}\text { Temperature, } \\
\text { major species }\end{array}$ & $\begin{array}{l}\text { Light inter- } \\
\text { ference from } \\
\text { stronger } \\
\text { processes }\end{array}$ \\
\hline & $\begin{array}{l}\text { Gas, possibly } \\
\text { solid, liquid }\end{array}$ & $\begin{array}{l}\text { CARS and } \\
\text { other non- } \\
\text { linear }\end{array}$ & $\begin{array}{l}\text { Temperature, } \\
\text { major species }\end{array}$ & $\begin{array}{l}\text { Very complex } \\
\text { experimentally. } \\
\text { Possibly very } \\
\text { sensitive. } \\
\text { Usable for tem- } \\
\text { perature in } \\
\text { somewhat dirty } \\
\text { environments. }\end{array}$ \\
\hline
\end{tabular}

(a) Adapted from Lapp 1980.

their primary benefit and justification is in providing data for the development and verification of models. The data that diagnostics provide are so specific that often they must be put into the framework of a model in order to have real value.

Generally the system to be modeled must be as well characterized as possible, which leads to efforts to use several tools to make several types of 
TABLE 5.3. Typical Scattering Cross Sections (a)

Scattering Process

Particle Scattering (Mie) $10 \mu m$ diam (elastic)

Particle Scattering (Mie) .1 um diam (elastic)

Atomic Fluorescence - visible (inelastic)

Molecular Fluorescence - simple molecules (inelastic)

CARS, other Stimulated, Nonlinear Processes (inelastic)

Rayleigh Scattering (elastic)

Rotational Raman Scattering (inelastic)

Vibrational Raman Scattering (inelastic)
Differential

Cross Section

$\left(\mathrm{cm}^{2} / \mathrm{st}\right)$

$10^{-7}$

$10^{-13}$

$10^{-13}$ to $10^{-18}$

$10^{-19}$ to $10^{-24}$

$10^{-20}$ to $10^{-30}$

$10^{-27}$

$10^{-30}$

$10^{-31}$

\section{(a) Adapted from Lapp 1980.}

measurements at once. Planning is then even more difficult, although the combinations are less expensive than separate efforts; for more useful information is being gained, and to some extent the different measurements provide checks on each other. Accuracy is exceedingly difficult to determine with most diagnostic systems, and systematic errors resulting from some experimental anomaly might not be noticed except in the effect on a different type of measurement. Increasing numbers of combined experiments (see Bibliography) and fully integrated experiment/modeling investigations have been developed. Laser diagnostics research is therefore an increasingly complex and expensive undertaking, yet truly important.

This report attempts to provide an understanding of laser diagnostics sufficient to provoke interest in the technology from the nonspecialist researcher. It emphasizes general features, shortcomings, and the need for targets of significance. The subject of target selection is developed further in a companion volume to this report. Applications of Laser Diagnostics in Energy Conservation Research (PNL-5281). The companion volume discusses some of the most advanced applications of diagnostics in several noncombustion fields and introduces some new ideas for further advances. These ideas are 
primarily targeted at the sponsor of the research, the Energy Conversion and Utilization Technologies Division (ECUT) of the Department of Energy. ECUT is responsible for developing the technology base for energy conservation through generic research. These two reports are an effort to enhance the effectiveness of ECUT's program in laser diagnostics, and to develop ideas and perspective for planners of additions and improvements to the program.

\section{S.1 REFERENCES}

Goulard, R. 1983. "Characteristic Parameters in Combustion Process and Their Accessibility to Current and Future Diagnostics." DOE/ER/05773-1, also DOE/ER/05773-2. U.S. Department of Energy. Available from National Technical Information Service, Springfield, Virginia.

Lapp, M. 1980. "Raman Scattering Measurements of Combustion Properties," in Laser Probes for Combustion Chemistry, American Chemical Society Symposium Series, Volume 134. American Chemical Society, Washington, D.C.

\section{S.2 BIBLIOGRAPHY}

Bechtel, J. M. 1979. "Temperature Measurements of the Hydroxyl Radical and Molecular Nitrogen in Premixed Laminar Flames by Laser Techniques." Applied Optics 18(13):2100-2106.

Dibble, R. W., W. Kollman and R. W. Schefer. 1984. "Conserved Scalar Fluxes Measured in a Turbulent Nonpremixed Flame by Combined Laser Doppler Velocimetry and Laser Raman Scattering." Combustion and Flame 55:307-321.

Fujii, S., M. Gomi and K. Eguchi. 1983. "A Remote Laser-Probe System for Velocity and Temperature Measurements." Journal of Fluids Engineering $105: 128-133$.

Sangiovanni, J. J., et al. 1983. "Optical Diagnostics for Diesel-Engine Applications." DOE/AL/17575-51, Argonne National Laboratory, Argonne, Illinois. 
CONTENTS

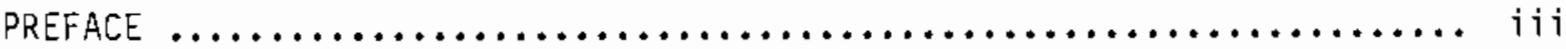

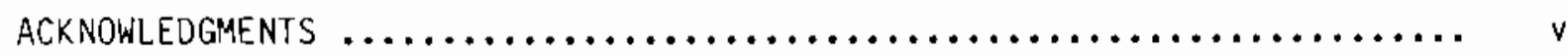

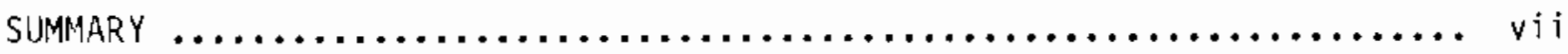

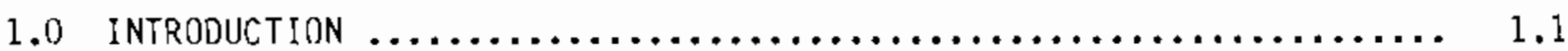

2.0 GENERAL CONCERNS IN USING LASER DIAGNOSTICS $\ldots \ldots \ldots \ldots \ldots \ldots \ldots \ldots .2 .1$

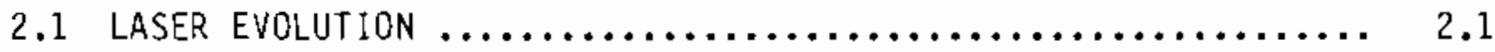

2.2 REQUIREMENTS FOR DATA REDUCTION $\ldots \ldots \ldots \ldots \ldots \ldots \ldots \ldots \ldots \ldots \ldots \ldots \ldots \ldots$

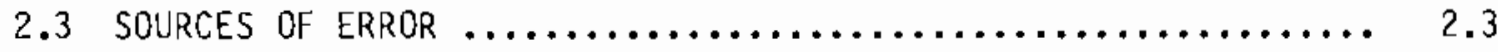

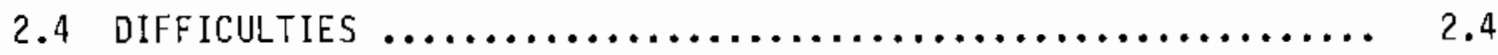

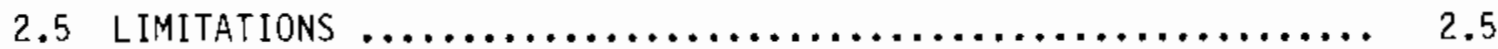

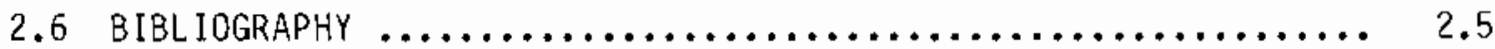

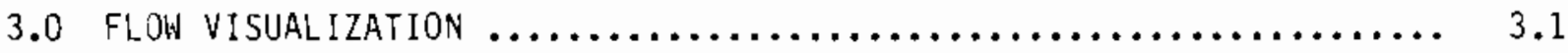

3.1 LASER SHADOWGRAPH AND SCHLIEREN PHOTOGRAPHY $\ldots \ldots \ldots \ldots \ldots \ldots .3 .2$

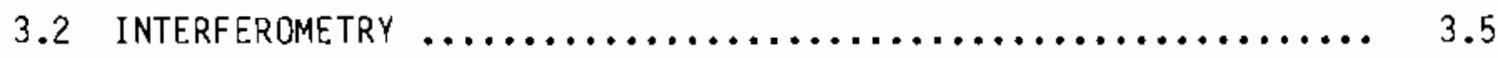

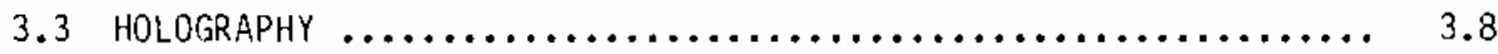

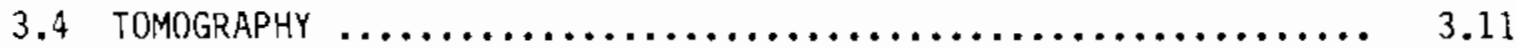

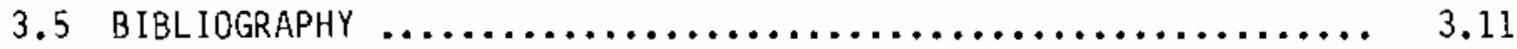

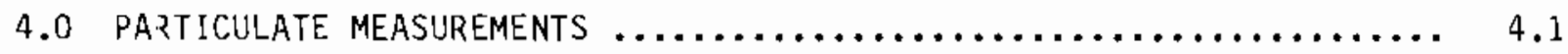

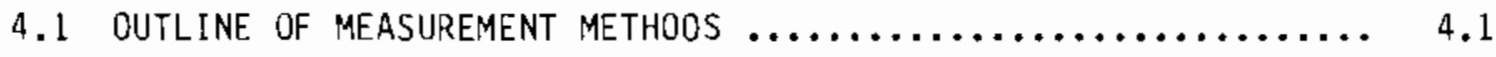

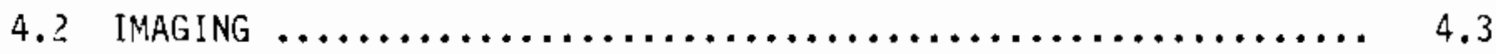

4.3 LIGHT SCATTER ING AND MIE THEORY $\ldots \ldots \ldots \ldots \ldots \ldots \ldots \ldots \ldots \ldots \ldots .4 .4$

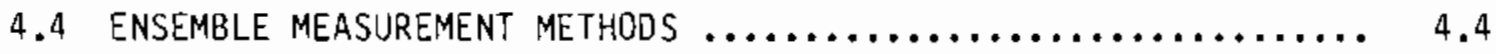

4.5 SINGLE-PARTICLE COUNTERS $\ldots \ldots \ldots \ldots \ldots \ldots \ldots \ldots \ldots \ldots \ldots \ldots \ldots \ldots$

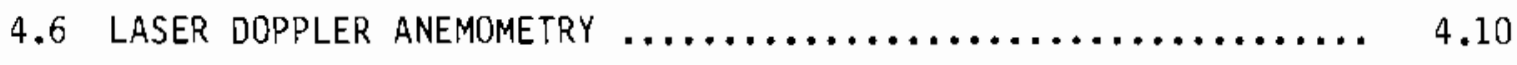




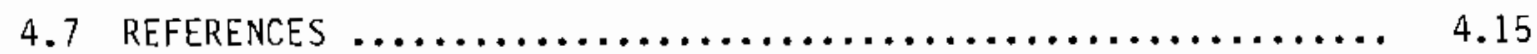

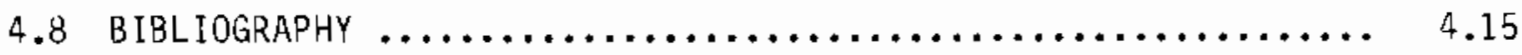

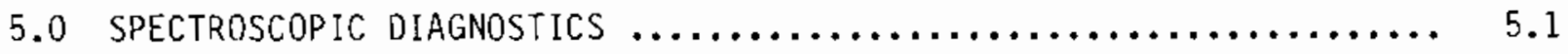

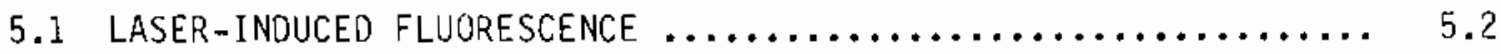

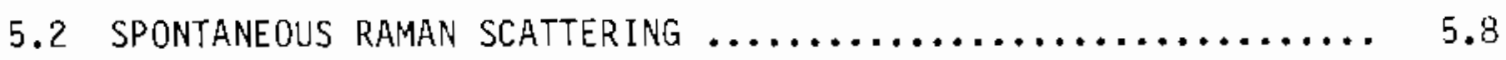

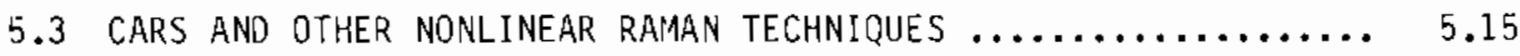

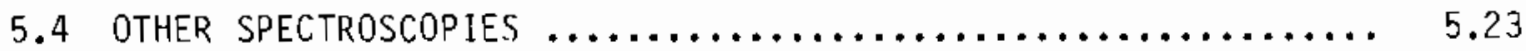

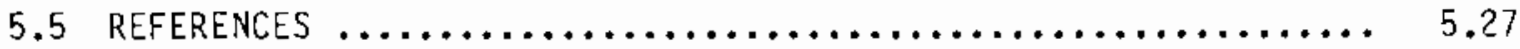

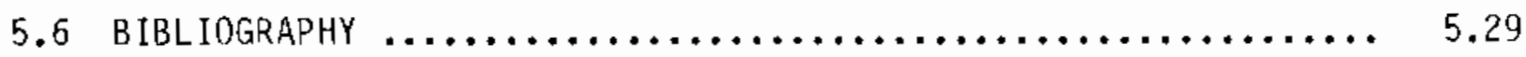




\section{FIGURES}

3.1 A Basic Shadowgraph System....................... 3.2

3.2 A Basic Schlieren System.......................... 3.2

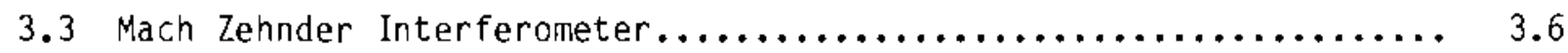

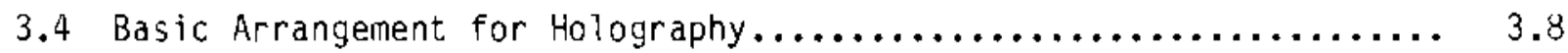

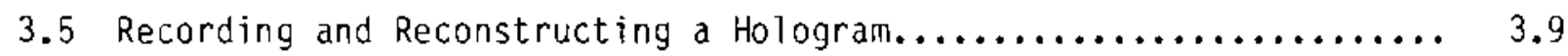

4.1 Particle Light Scattering......................... 4.5

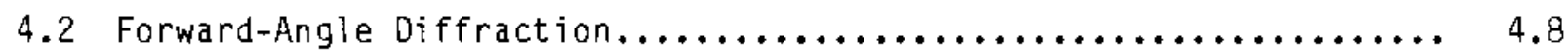

4.3 General Configuration for Single-Particle Counting............ 4.9

4.4 Basic Arrangement for Laser Doppler Anemometry.............. 4.11

4.5 Fringe Interpretation of LDA Measurements................ 4.12

5.1 Basics of Laser-Induced Fluorescence Measırements............ 5.3

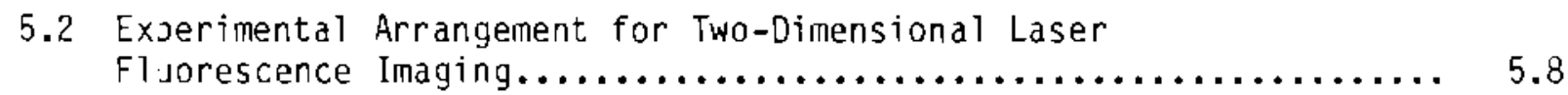

5.3 Outline of Basic Raman Transitions..................... 5.10

5.4 Rainan Spectroscopy of a Surface..................... 5.11

5.5 Basics of CARS Measurement.............................. 5.17

5.6 Tysical CARS Experiment Schematic..................... 5.21

5.7 Use of CARS Spectrum for Temperature Measurements ............ 5.22

5.8 Exjerimental Arrangement for Photothermal Deflection
Spectroscopy $\ldots \ldots \ldots \ldots \ldots \ldots \ldots \ldots \ldots \ldots \ldots \ldots \ldots \ldots \ldots \ldots \ldots \ldots \ldots \ldots \ldots \ldots \ldots \ldots$ 
TABLES

S.1 Laser Visualizations............................... viij

S.2 Light-Scattering Diagnostic Methods................... ix

\$.3 Typical Scattering Cross Sections......................

4.1 Size Ranges of Particle-Sizing Techniques................ 4.2 


\subsection{INTRODUCTION}

During the past decade, intensive research and development activities have demonstrated the feasibility, checked the accuracy, and extended the sensitivity of laser diagnostics for combustion systems. Combinations of diagnostics are now capable of in-situ, time- and space-resolved measurements of temperature, velocity and species concentration. Improvements in diagnostic systems are resorted regularly, driven by developments in laser and detector technology and increased levels of work in the field.

Laser diagnostics are now mature enough to be truly useful in combustion research. Although the techniques can be both expensive and difficult, they are the keys to solving the critical problems facing combustion researchers today. These problems center on the need for more in-depth understanding of combustion mechanisms. Many related areas within the field of energy conversion demand in-situ measurement as well. A wide variety of combustion and noncombustion measurements are needed to help build and validate process models to be used in design and control of energy conversion systems. The lack of effective models is a primary limitation in the effort to promote energy conservation through increased conversion system efficiency.

Successful application of laser diagnostics can be expanded in two ways. The first is to broaden the "user base" by introducing nonexperts and even student researchers to diagnostics capabilities in such a way that they can determine the merit of using diagnostics in their own work. This might be done by providing user-oriented information on the strengths and limitations as well as the physical basis for each method. Potential users could then begin the process of applying a technique to a problem by bringing their problem to the attention of diagnosticians. The second approach is to search specifically for applications based on important research needs and current diagnostic capabilities. Such an approach is especially useful in noncombustion areas where the tools have received less publicity. 
The Pacific Northwest Laboratory (PNL) (a) has contracted with the U.S. Department of Energy (DOE) to study the application of laser diagnostics to novel and nontraditional problems. The investigation was developed for the Energy Conversion and Utilization Technologies Division (ECUT), which conducts long-term research in processes and materials in order to strengthen the technology base for energy conservation. The program's trailblazing use of laser diagnostics in internal combustion engines to probe in-cylinder processes is an example of ECUT's basic, yet pragmatic, approach. There are many other important problems in energy conversion that might benefit from the improved understanding and modeling available through diagnostics, but the costs and benefits of such undertakings are not clear. The goals of the current PNL investigation are to provide perspective and further ideas for ECUT research programs.

PNL's approach to the project has been to focus on the development of application ideas. This effort has taken place as outlined above, both through PNL's own efforts and through stimulating researcher involvement in a wide variety of important problem areas. The results of the work are presented in two volumes. One volume, Applications of Laser Diagnostics in Energy Conversion Research, discusses several new and developing diagnostics applications. This volume presents a relatively elementary discussion of a wide range of diagnostics, with special attention to the physical basis for the techniques and their fundamental and generic limitations. It is intended to provide sufficient insight into diagnostic tools to stimulate researchers as well as to educate students and others. It emphasizes better-established methods, since the state of the art in laser diagnostics is constantly changing.

A11 the optical diagnostic methods involve light and its interactions with combustion components and flowfields. The techniques vary considerably in cost, complexity, and the type and quality of information they provide. In the report, general concerns--including the difficulties and limitations of using diagnostics--are outlined first. Then, the methods are presented in three

(a) PNL is operated for the U.S. Department of Energy by Battelle Memorial Institute. 
groups (in increasing order of complexity): flow visualization, particle measurement, and spectroscopic methods. These groups overlap somewhat--visualization may be used for measuring particle size, for instance. The basic thrust of effort within each group, however, is consistent, so each group is placed in a separate chapter. 



\subsection{GENERAL CONCERNS IN USING LASER DIAGNOSTICS}

There are several general aspects of laser diagnostics and their performance that are important but are not discussed at length for each tool. Instead, they are covered here and referred to in later discussion. Occasional points made in this chapter refer (as examples) to aspects that will be discussed in subsequent chapters, and may involve new terminology for the reader. Review of such matters early in the report is necessary, because these considerations are important in assessing the use of nonintrusive optical techniques. The laser diagnostics discussed in this report comprise a very diverse set, however, and the potential user must be attuned to his specific application as well as the generalities of the whole class of techniques. Optical diagnostics work has been proposed for the measurement of almost every flow parameter in a wide variety of systems, ranging from duct flows to free shear flows, and from the clear to highly particle-laden. Often, however, the results are hardiy more valuable than those obtainable with conventional intrusive techniques. Important characteristics relevant to the selection of laser diagnostics are discussed here, including lasers and data reduction equipment, experimental error, difficuities, dangers, and limitations of present techniques.

\subsection{LASER EVOLUTION}

Most of the basic principles used for optical investigations of fluid dynamic or combustion phenomena were established fairly early in this century. Even the simpler spectroscopic phenomena, Raman scattering and molecular fluorescence, were well enough understood by midcentury to allow a variety of chemical measurements. Our knowiedge of the spectroscopy of most molecules is based on data accumulated over many years of research, much of it without use of lasers. In a similar manner, much of our current knowiedge of fluid mechanics was developed with the aid of flow visualizations utilizing conventional light sources. Once lasers became available in the early 1960s, their potential as light sources for diagnostics was apparent. Their high intensity, coherence, monochromaticity, and potential for extremely short pulse lengths gave new life to many older techniques. Several new techniques were created as 
well, including laser Doppler anemometry (LDA) and coherent anti-Stokes Raman spectroscopy (CARS), which essentially enabled the accomplishment of in-situ work.

Much of today's in-situ spectroscopic work requires, not "a laser," but sophisticated, high-powered, tunable lasers. The instability and general cantankerousness of such devices are almost legendary, and add considerably to experimental difficulty. If the field continues to evolve, the lasers may be improved beyond today's adaptations of military specifications. Perhaps they will even be developed into "hardened" systems, relatively immune to bumps and temperature variations. Lasers are the key to many of today's most successful diagnostic procedures, but they add an extra field of knowledge to those that the successful diagnostician must master.

\subsection{REQUIREMENTS FOR DATA REDUCTION}

Laser diagnostics vary considerably in their data reduction requirements, the magnitude of their requirements generally being indicative of their overal1 complexity. The older flow visualization methods still use film to record images, and since they do not provide much quantitative information, they do not require much computation to evaluate inferred flow properties. Where more quantitative potential exists, however, such as with laser holograms, researchers are investigating the use of image-processing techniques in concert with video cameras or other electronic imaging systems. Unfortunately, this requires substantial investment in computer equipment.

Particle-sizing methodologies have a wide range of computational requirements, varying from the minimal demands of imaging methods (as currently practiced) to the considerable calculation necessary for evaluating Mie scattering at different angles or for deconvoluting laser intensity and particle-sizing effects on a given measurement. Some methods require relatively fast electronics for data acquisition as well, and such electronics are still an art in most applications.

Finally, spectroscopic methods all depend heavily on computation, at least to gain any sort of efficiency in operation. Considerable effort is involved 
in developing temperatures or concentrations from measurements of light intensities at various frequencies. A substantial amount of previously acquired information is necessary on spectra, excited-state quenching rates, extinction coefficients, optical theory, and so on. Some techniques or situations (or molecules) require considerably more complex computations than others. The complexities increase the difficulty (and thus the planning effort), the expense, and the error of a given measurement. One fortunate aspect of the tremendous amount of data reduction involved in laser diagnostics is that it can still be relatively inexpensive. Computers are inexpensive compared with the cost of laser gear, and can sometimes be used to compensate for the shortcomings of the remaining hardware. In addition, many of the more difficult programs used for data reduction are available from various organizations at minimal cost.

\subsection{SOURCES OF ERROR}

Many causes of uncertainty exist in a given measurement, in addition to inaccuracies, incompleteness, or bias in a data reduction methodology. These uncertainties are very practical considerations, which can either make a measurement impossible or simply provide a certain level of error. Such considerations include interferences, system perturbations, and signal transmission variations. Interferences are generally the most serious of these possibilities. Background luminosities, laser-induced particle fluorescence or lasermodulated particle incandescence, and particle (Mie) scattering can totally swamp meaningful signals. Window fluorescence and spurious laser scattering can also cause difficulties.

Such system perturbation considerations as medium heating or optical breakdown effects set upper limits on allowable laser focal fluxes. Signa? transmission concerns include lens damage, soot extinction and beam steering and defocusing. Fluxes through lenses and other optical components must be limited to prevent damage. Soot extinction (and other particle extinction) can make many types of measurements impossible in actual combustors. Beam steering (due to strong density gradients in a flow) is a potentially serious problem in 
larger devices, especially for interference-based methods such as LDA and holography or for beam-mixing procedures such as CARS.

Many of the limitations listed here determine whether a measurement is possible at all. Others, like beam steering, may only degrade the measurement to an unquantifiable degree. For many of the laser diagnostics it is difficult or impossible to grasp the error component of a given measurement, since the laser probes can be used where other methods cannot; thus there are no checks or calibrations. Comparisons have generally been made (in simple environments), but the resolution of such measurements seldom permits exact judgments. Accuracy is often interrelated in complex ways with a tool's resolution and range, and compromises must often be made among these because of low signal levels. Resolution in time and space, as well as the tool's measurement range, are then based on a lower limit for the number of photons needed to produce a viable signal. An attempt is made here, in the discussions of several of the tools, to examine ways in which these restrictions and relationships affect tool performance.

\subsection{DIFFICULTIES}

The attempt to find a route through the practical considerations listed above, many of which may apply for a given measurement, is the major source of complication in laser diagnostic measurements. Certain types of difficulties appear to be endemic, particularly to the multiple beam measurements like LDA and CARS, and spectroscopic measurements like laser-induced fluorescence ( $L$ IF), Raman, and CARS. For example, the former are difficult to align and focus in an optimal manner. The latter, except for the simplest of measurements (e.g., $\mathrm{OH}$ radicals with $\mathrm{LIF}$, require a storehouse of spectroscopic data that of ten must first be found experimentally. Once found, such data can on occasion conclusively show that a particular measurement is impossible.

The spectroscopic methods and LDA generally require a great deal of planning in order for a measurement to be successfully executed in a new environment. The tool and laser-tuning strategy must be chosen, and the data reduction plan and program defined. Finally, even with an experiment running smoothly, there is an element of danger involved. Many experiments include 
high-powered lasers, combustible fuels, flames (and thus high temperatures) and toxic exhaust gases; therefore, considerable extra expense and complexity are created to ensure safe operation.

\subsection{LIMITATIONS}

Limitations on laser diagnostics are generally the result of complex interplay between a large number of factors. Some general matters have been reviewed in the preceding paragraphs, including the quirks of high-powered lasers, the need for involved data reduction, and the vast array of considerations that must be addressed in working up a diagnostic approach. These limitations will probably never disappear; they will, however, become more familiar and thus less difficult. Much of the effort in diagnostics development is not toward making current methods easier, but rather toward extending their capabilities. The goal of this development is to increase sensitivity to variables of greater physical importance, and its success has been substantial. It has not, however, left solidly established methods with known limitations for those not interested in furthering the state of the art. Since that state is in constant flux, this report emphasizes the better-known methods.

\subsection{BIBL IOGRAPHY}

A number of documents have been prepared describing experiences with and advances in diagnostics; many of them are listed here. These documents are themselves valuable sources of references, particularly the efforts of Bechtel, Dasch and Teets and the recent assessment edited by Hardesty.

Bechtel, J. H. and A. R. Chraplyvy. 1982. "Laser Diagnostics of Flames, Combustion Products, and Sprays." Proc. of the IEEE 70(6):658-677.

Bechtel, J. H., C. J. Dasch and R. E. Teets. 1983. "Combustion Research with Lasers." General Motors Research Publication GMR-4402, also a chapter in Laser Applications. Academic Press, New York, New York.

Crosley, D. R. 1980. Laser Probes for Combustion Chemistry. ACS Symposium Series 134, American Chemical Society, Washington, D.C.

Eckbreth, A. C. 1981. "Recent Advances in Laser Diagnostics for Temperature and Species Concentration in Combustion." Eighteenth Symposium (International) on Combustion. The Combustion Institute, Pittsburgh, Pennsylvania. 
Eckbreth, A. C., P. A. Bonczyk and J. F. Verdieck. 1979. "Combustion Diagnostics by Laser Raman and Fluorescence Techniques." Prog. Energy Combustion Sci. 5:253-322.

Greenhalgh, D. A. 1983. "Gas Phase Temperature and Concentration Diagnostics with Lasers." International Conference on Combustion in Engineering (Vol. 1). Institution of Mechanical Engineers, London, England.

Hardesty, D. R. 1980. "Diagnostics and Instrumentation Requirements for Advanced Power Systems." ASME Paper 80-GT-129, American Society of Mechanical Engineers, New York, New York.

Hardesty, D. R., ed. 1984. An Assessment of Optical Diagnostics for In-Situ Measurements in High Temperature Coal Combustion and Conversion Flows. 84-8724. Sandia National Laboratories, Livermore, California.

Lapp, M. 1975. "Optical Diagnostics of Combustion Processes." SPIE Volume 61 (Energy Conversion). 42-50.

Lederman, S., and S. Sacks. 1984. "Laser Diagnostics for Flowfields, Combustion, and MHD Applications." AIAA Journal 22(2):161-173.

Moore, C. B., ed. 1979. Chemical and Biological Applications of Lasers. Academic Press, New York, New York.

Penner, S. S., ed. 1983. "Assessment of Research Needs for Coal Utilization." DOE Coal Combustion and Applications Working Group (CCAWG), U.S. Department of Energy Fossil Energy Division, Washington, D.C.

Schwar, M. J. R., and F. J. Weinberg. 1969. "Laser Techniques in Combustion Research." Combustion and Flame 13:335-374. 


\subsection{FLOW VISUALIZATION}

Various methods for visualizing and photographing flows in combustion and compressible aerodynamics have been developed during the last century. A number of these methods use optical phenomena, many of which have been implemented with lasers in recent years. In many cases the use of a laser does not bring about dramatic improvements; indeed, sometimes the image quality deteriorates with laser light. However, the power, monochromatic nature, high temporal and spatial coherence, and short pulse abilities of lasers now make visualization possible in new situations. Bright transient combustion flows are among the most important of these.

The major methods to be discussed in this chapter include shadowgraphs, schlieren, interferometry, holography, and tomography. The first three of these are based on index-of-refraction information. The first two are very similar and will be discussed together. Moire deflection mapping, another method based on ray deflections due to density (and index-of-refraction gradients), will not be discussed here. For schlieren, shadow and Moire, a laser may or may not be used as a light source. Often, however, the experimenter is interested in using the technique to capture the time dependence of a dynamic event such as an explosion. In such a case, pulsed lasers are preferred light sources. Interferometry is another method of recovering index-of-refraction information using light's wavefront phase changes. Holography, which is also interferometric, is a method of recording both intensity (as is normally done) and phase of light that gives complete information. It can be used to record shadow and schlieren visualizations, to record interferograms, and even to size particles. Holography does require a laser, however, because monochromatic, coherent light is needed. Finally, tomography is a method of combining lineof-sight toois to give spatial resolution. It can be used for absorption as well as visualization techniques, but as it is not widely applied, it is oniy briefly mentioned in this report. 


\subsection{LASER SHADOWGRAPH AND SCHLIEREN PHOTOGRAPHY}

Shadow and schlieren methods have been used for a century to better understand such dynamic phenomena as explosions, sparks, shock and sound waves, turbulence, air flow around projectiles, and flame propagation. To fit these diverse flows, many arrangements of lenses and mirrors are possible (Figures 3.1 and 3.2). Some of the flows are clearly visible (e.g., flames) but regions of interest (e.g., the flame front) are sometimes better visualized with shadow or schlieren methods.

\section{Background}

Both shadow and schlieren systens are relatively simple and are based on the effect of density gradients on light. A change in the density implies a change in index of refraction, which deflects light. Light originally perpendicular to the flow is deflected toward regions of higher density and larger

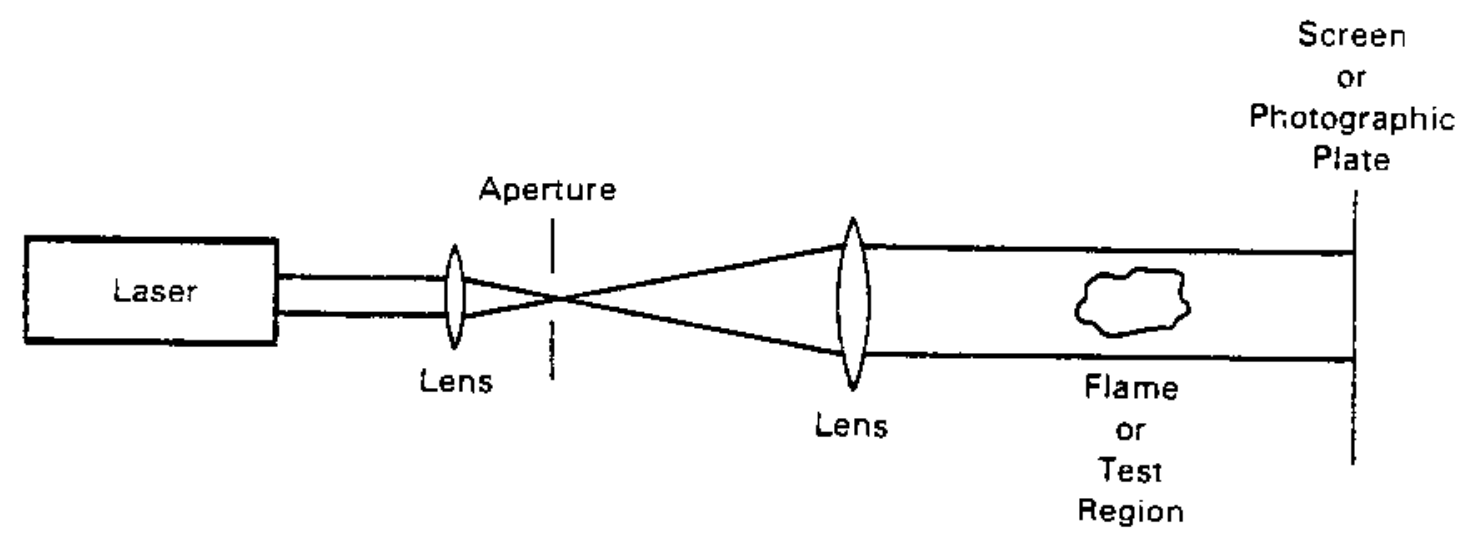

FIGURE 3.1. A Basic Shadowgraph System

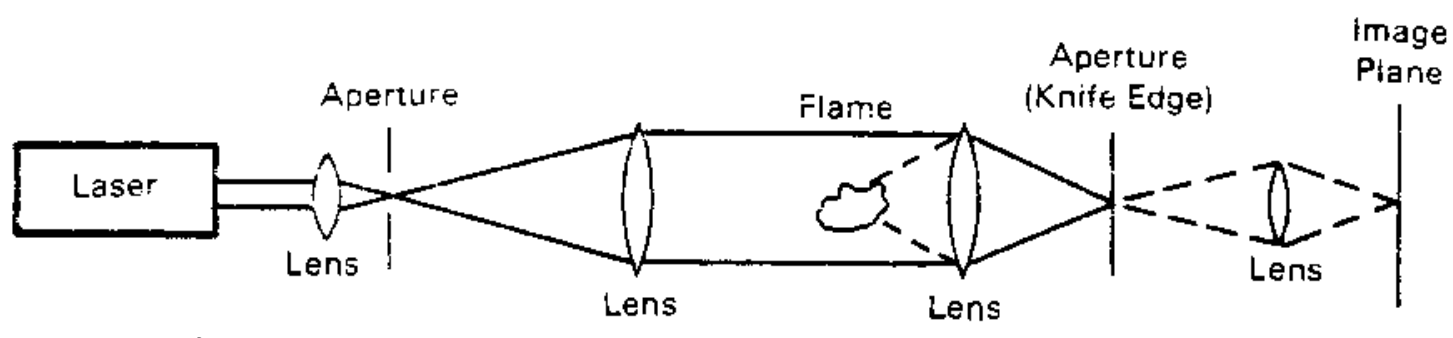

FIGURE 3.2. A Basic Schlieren System 
index of refraction. A uniform index provides no shift; a uniform index gradient provides the same shift for all the light. Shadows are formed, therefore, by a changing gradient of the refractive index, i.e., by the second derivative of the index, and thus of the density of the flow. The shadowgraph is ideal for visualization of flow structures that have very rapid changes in their gradients.

In schlieren systems, a knife edge, slit, hole or other type of stop is used to block either deflected or undeflected light coming from the test region. If the deflected rays are blocked, they do not reach the screen (as they do in a shadowgraph) and are seen as dark regions on the bright screen background. Thus, the image depends on the deflection (the first derivative of density) rather than the changes in deflection (the second derivative of density). Because of this, schlieren methods are generally more sensitive than shadowgraphs. The physical setup for schlieren simply adds the extra knife edge or stop to the shadowgraph arrangement.

Conditions

The practical requirements for successful schlieren or shadow experiments are similar, with one extra condition for schlieren systems.

- The light used should be well collimated and pass through the test section as a parallel beam.

- Test sections must be planar or rotationaliy symmetric and transparent.

- Optical components should be of good quality, particularly for schlieren systems. The use of physically large test sections suggests the use of mirrors rather than lenses in the systems, as large accurate lenses are very expensive--if available at all.

- For transient flows, short light pulses are required, which are generally easier to obtain with lasers than with spatially filtered st.robes. 
- For luminous flows, light should be monochromatic so that unwanted light can be filtered out with a narrow band-pass filter. This is best achieved with a laser.

- In schiieren systems, proper alignment with the knife edge or stop is required, so vibration must be avoided. This is generally not such a problem with shadowgraphs.

\section{Difficulties}

Shadow and schlieren systems are fundamentally simple and therefore fairly easy to use. Except for discernment of geometric flow features, however, they are only qualitative. This is partiy because of limitations in the quality of optical components, the light source used, image displacement, and (especially for schlieren systems) alignment and vibration-proofing. Before these weaknesses are discussed, the implications of being restricted to planar or rotationally symmetric flows must be mentioned. Most practical flows are not this simple; nor are many practical flows transparent. Despite their simplicity, therefore, shadow and schlieren systems are generally limited to laboratory applications in special test sections.

Although shadow and schlieren methods are basically qualitative, attempts have been made to integrate image intensities to find fiow densities. Success has been slight, partly because of the limitations noted above and partly because of nonlinearities introduced into the process by the film on which the image is stored. The varying density gradients portrayed by schlieren can even be displayed in color, but this color-coding does not provide accurate density.

Some of the reasons for the low accuracy are image displacement (for both methods), speckle (for laser systems), and misalignment (for schlieren). The image displacement is of course unavoidable, as it is caused by the bending of the light rays that also generates the visualization. "Speckle" is the optical term for such coherent noise as diffraction inages created by dust, marred mirrors, or other imperfections in the optical system. Speckle photography is itself an imaging method, but it is just a probiem in schlieren and shadow systems. Misalignment is a problem with schlieren systems because the light must be properly focused on the knife edge to provide a good image. Laser 
systems can be focused to a very fine spot, but with puised systems in particular it is more difficult to set and keep the alignment.

Current Work

Laser shadowgraph and schlieren systems have been used in numerous aerodynamic and combustion flow experiments of a relatively practical nature. Shadowgraphs have been used in a variety of in-cylinder engine experiments to visualize intake air motion, swirl, flame propagation, and flame wrinkling. Schlieren systems have been used to study intake flows, fuel air interactions, and flames in a variety of engines. Both methods have their origins in work with airfoils and ballistics and other high-speed flow phenomena, where they are still applied.

\subsection{INTERFEROMETRY}

Like the shadow, schlieren, and deflection mapping methods, interferometry provides index-of-refraction information. Unlike shadow and schlieren methods, however, interferometry yields the index of refraction (or density) itself, although some mathematical data reduction is necessary. Lasers brought considerable improvements to interferometry because of their coherent light, and made operation easy as well. A variety of possible interferometric arrangements exists, but the most prevalent has been the Mach-Zehnder type (i11ustrated in Figure 3.3).

\section{Background}

Interferometry is based on light wavefront phase changes. In the method, a test and a reference beam emanating from the same laser interfere with one another, generating a pattern of straight interference fringes. If the originally uriform density distribution in the test beam is altered, the originally straight and uniform interference pattern also will change. Each fringe represents a region of constant phase. Because the phase shift is an integral effect of the index of refraction along the optical path, measurements are essentially limited to planar or radially symmetric systems. 


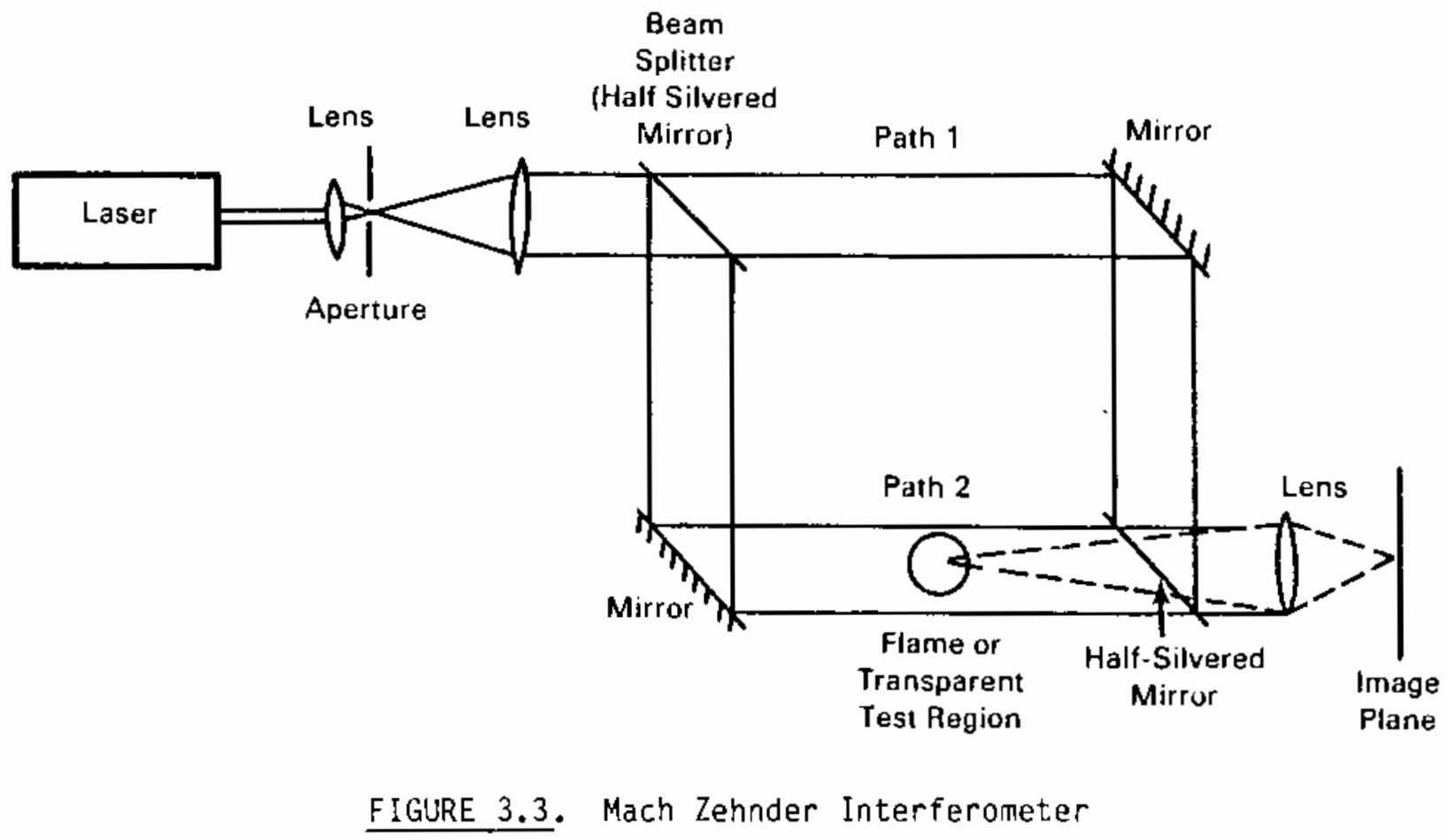

Conditions

Several requirements must be met to produce acceptable Mach-Zehnder interferograns:

- High-precision optical and mechanical components are necessary, and provision made for constant uniform temperature.

- Alignments and adjustments must be carefully set and maintained and vibrations avoided to maintain sensitivity.

- For pulse lasers, optical path lengths must be approximately the same (exact restriction depends on pulse duration). For continuous lasers, this is less critical.

- Light must be parallel, which is readily obtained with a laser.

- Approximately plane or radially symnetric flows are necessary in order for relatively accurate measurements to be made.

- In high-temperature systems, care must be taken to pick a laser frequency that will not be absorbed by the gas. 


\section{Difficulties}

The difficulties with interferometric measurements usually involve attempts to get clear fringes from nonideal optics and flows. The ideal situation is infinite width fringes from exactly parallel beams through perfect optics and planar flow. The difficulties arising from imperfection involve increasing complexity in data reduction. In practical systems, the fringes are quite fuzzy. Positive or negative density excursions cannot be distinguished. It may also be difficult to identify the same fringe on each side of a discontinuity such as a shock wave. Speckle (coherent noise) makes fringes even more ragged, and often makes ordinary light preferable to lasers. If the flows being investigated are not planar, inversions must be performed on the basis of assumptions about the symmetry of the distribution of the index of refraction. These inversions can be very complex and are practically limited to the case of radial symmetry.

\section{Current Work}

Interferometers have been used to study flame ignition and growth in various systems. Sometimes they are used to find spatial temperature profiles (based on density). Examples include work on flame spread over surfaces and ignition investigations of a variety of substances.

\section{Other Interferometers}

Many different interferometric systems have been demonstrated using both ordinary and laser light. There is a combined schlieren/interferometric technique, using polarized ordinary light, called a "schlieren interferometer". Another method that utilizes diffraction gratings to generate test and reference beams can occasionally be easier to use than Mach-Zehnder setups. Another arrangenent, the Shearing interferometer, makes use of reflections from the front and rear surfaces of a mirror. It may provide similar operational benefits, along with component cost reductions, but it is less sensitive. Finally, a "multiple beam interferometer" is a seldom-used tool that can provide extremely sharp fringes with a simple apparatus by using partially silvered mirrors to pass light beams through the test section many times. 


\subsection{HOLOGRAPHY}

Holography is a method for recording light's phase and intensity relationships simultaneously (see Figure 3.4). It can be used for particle sizing and ordinary photographic flow visualization (when there are particles in the flow large enough to generate their own photographic "inages"). The method provides excellent depth of field in such applications. When there are no particles in transparent flows, schlieren or shadowgraph methods can be used in combination with holographic recording. Finally, holographic interferometry has enjoyed considerable application, including combustion research, and will be emphasized here.

\section{Background}

A hologram is created by recording the interference pattern from combining a reference and an object beam (which passes through the flow) split from the same original coherent laser beam. This pattern contains both intensity and

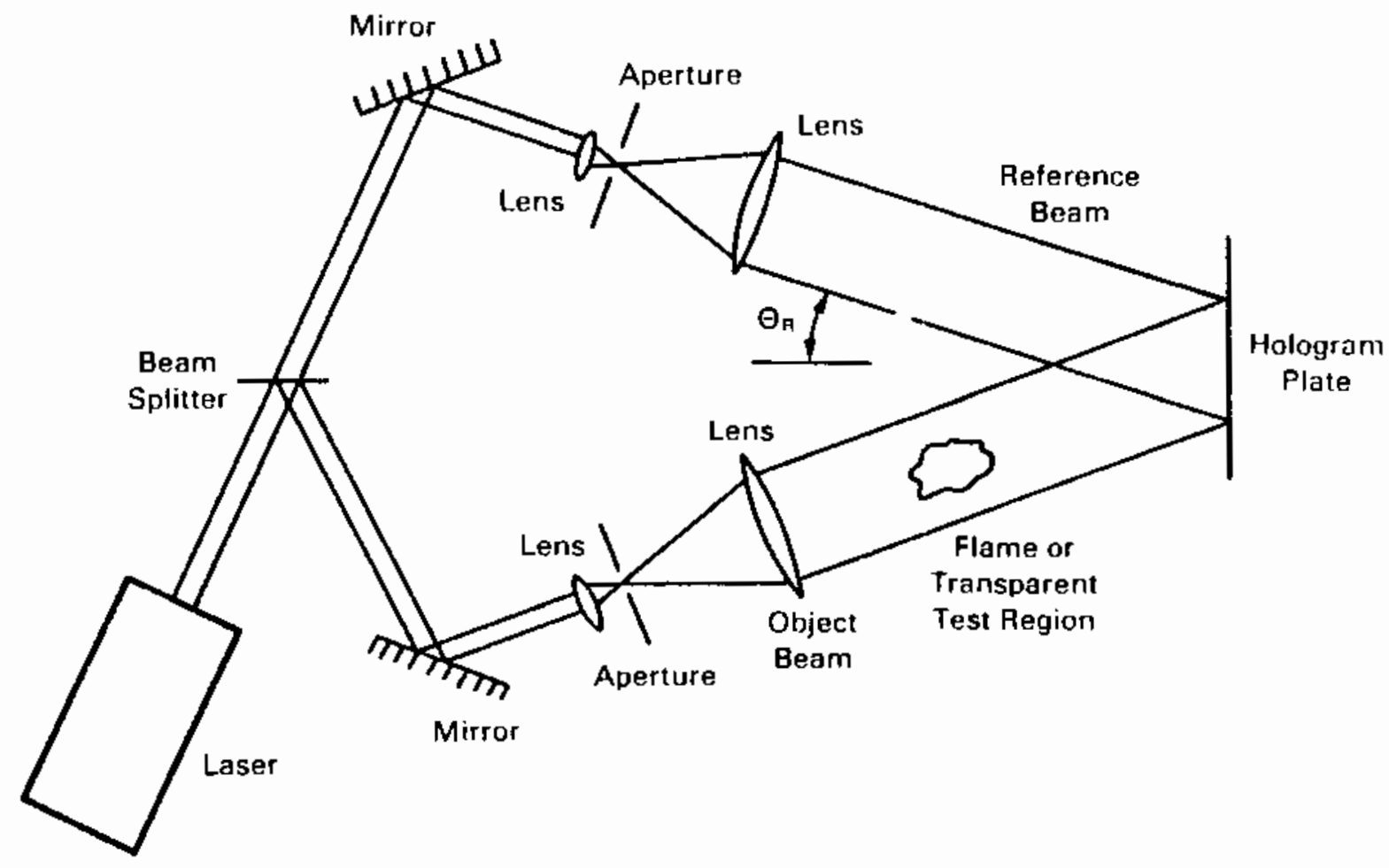

FIGURE 3.4. Basic Arrangement for Holography 
phase information. Figure 3.5 illustrates the recording and the reconstruction of a hologram. If the same optics are used for reconstruction, the error due to optical imperfections cancels out, which is highly advantageous. Velocity measurements in three dimensions can be made by taking several exposures of a particle-containing flowfield at precise time intervals. If no particles are available or desired, a schlieren or shadow arrangement can be used to record density derivatives.

Tre most important application of holography is in interferometric measuremerts. This type of interferometry is not subject to the restrictions of symmetric density fields that had to be made for intensity-only methods. Two methods are used. Double-exposure holographic interferometry uses two sequential exposures on the film plate: the first is made in the absence of the flame or flow (or object) under study, and the second is made with it in

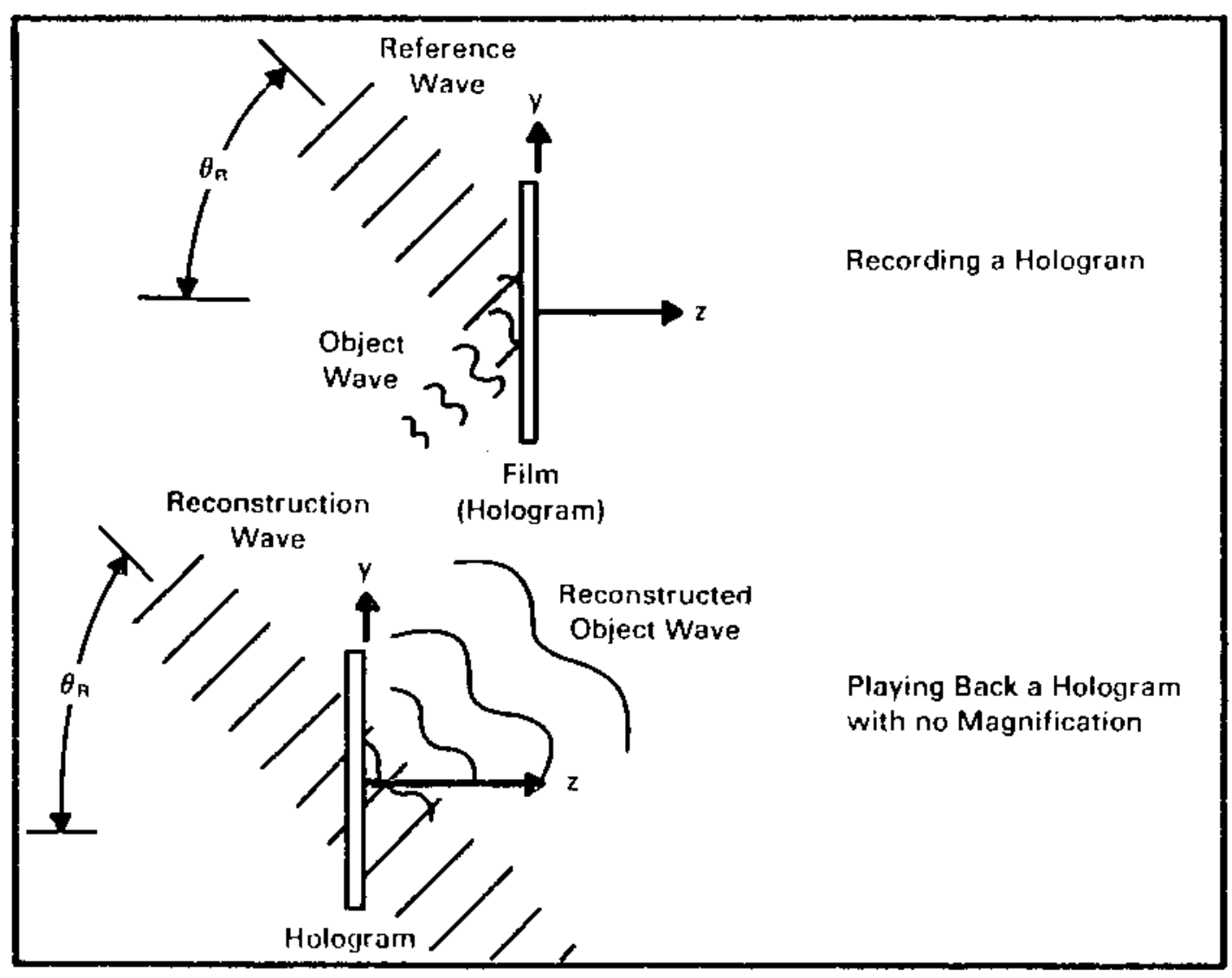

FIGURE 3.5. Recording and Reconstructing a Hologram 
place. The fringe patterns recorded correspond to many different viewing directions through the flame, like many different Mach-Zehnder interferograms recording different flame orientations. Real-time measurements are made possible by mounting a developed hologram (without the flame) in its initial orientation and re-illuminating it with test and reference beams with the flame on. Interference will be observed, and any changes that occur in the test section as a result of the flame can be photographed as they occur. Conditions

To make and use a successfui hologram, the following conditions must be met:

- The laser light used must have a sufficient coherence length. This puts certain restrictions on the optical path lengths used, particularly with pulse lasers.

- Syster alignment is important, especially when the hologram is being reconstructed.

- The medium under investigation must not be optically dense.

- The method cannot be used to investigate large volumes because that would require large, very accurate lenses at very high prices.

Difficulties

Various difficulties are associated with holography, generally depending on the type of measurement being made. All holographic methods require considerable experimental skili. One universal requirement is the stability of optical positions, since small component displacements cannot be distinguished from changes in the flow being investigated. This is particularly important for interferometry, and is a source of major difficulty in real-time measurements where the developed hologram must be very accurately repositioned.

There are other significant difficulties with interferometry as well. It is usualiy not possible to observe fringes from all directions; only a few observation conditions are usable. The evaluation of a density field from the finished hologram is laborious, and requires special computer codes. Resulting accuracies vary considerably and are not easy to estimate. 
For velocity measurements, particles are needed in the flow under investigation. Particles must be well distributed and in the proper size range (about 0.1 min). Unfortunately, particies this size can deviate considerably from streamlines, especially in curving or low-density flows. For this reason laser Doppler techniques, which can measure velocity in any direction but only at one spot, are usually preferred.

Current Work

Holographic interferometry is an important method of studying small-dimensional changes (e.g., due to vibration) that occur in engineering development applications. It has also been used in certain combustion work as an extension of ordinary interferometry, measuring flame speeds and temperatures, and even air-fuel ratios. Extraneous fringes from windows and other anomalies are cancelled in a double-exposure measurement, which has proved to be a useful benefit. Finally, holographic interferometry, which does not require symmetry assumptions in order to provide point information, has been used to check the effect of such assumptions in ordinary interferometry in flames.

\subsection{TOMOGRAPHY}

Tomography is a formalized approach to the use of inversions (as mentioned under "interferometry") to produce spatial information from line-of-sight data. The method takes advantage of technology developed for X-ray medical diagnostics. Measurements are made along parallel, evenly spaced lines from several directions, forming a data set from which concentrations or temperature distributions can be reconstructed. The method has been used primarily for absorption measurements, a simple and well understood method by itself. Tomography is most readily applied to flow fields with axisymetric properties.

\subsection{BIBLIOGRAPHY}

General references and additional techniques:

Farrel1, P. V., and D. L. Hofeldt. 1984. "Temperature Measurement in Gases Using Speckle Photography." Applied Optics 23(7):1055.

Keren, E., et al. 1981. "Measurements of Temperature Distribution of Flames by Moire Def lectrometry." Applied Optics 20(24):4263-4266. 
Merzkirch, W. 1974. Flow Visualization. Academic Press, New York.

Weinberg, F. J. 1963. Optics of Flames. Butterworth's, London, England.

Shadow and Schilieren Methods:

Davies, T. P. 1981. "Schlieren Photography--Short Bibliography and Review." Optic and Lens Technology 13.

Johnston, S. C. 1980. "Raman Spectroscopy and Flow Visualization Study of Stratified Charge Engine Combustion." SAE Paper 800136, Society of Automotive Engineers, Warrendale, Pennsylvania.

Meintjes, K., and A. C. Alkidas. 1982. "An Experimental and Computational Investigation of the Flow in Diesel Prechambers." SAE Paper 820275, Society of Automotive Engineers, Warrendale, Pennsylvania.

Sinnamen, J. F., D. R. Lancaster and J. C. Steiner. 1980. "An Experimental and Analytical Study of Engine Fuel Spray Trajectories." SAE Paper 800135 , Society of Automotive Engineers, Warrendale, Pennsylvania.

Smith, J. R. 1982. "The Influence of Turbulence in Flame Structure in an Engine." SAND 82-8722, Sandia National Laboratories, Livermore, California.

Witze, P. 0., and F. R. Vilchis. 1981. "Stroboscopic Laser Shadowgraph Study of the Effect of Swirl on Homogeneous Combustion in a Spark-Ignition Engine." SAE Paper 810226, Society of Automotive Engineers, Warrendale, Pennsylvania.

Zhang, N., and W. J. Yang. 1983. "Visualization of Evaporative Convection in Minute Drops by Laser Shadowgraphy." Review of Scientific Instruments $54(1): 93$.

\section{Interferometry:}

Maly, R., and M. Vogel. 1979. "Initiation and Propagation of Flame Fronts in Lean $\mathrm{Ch}_{4}$-Air Mixtures by the Three Modes of the Ignition Spark." Seventeenth Symposium (International) on Combustion, Pp. 821-831. The Combustion Institute, Pittsburgh, Pennsylvania.

Mehta, J. M., and W. M. Worek. 1984. "Analysis of Retraction Errors for Interferometric Measurements in Multicomponent Systems." Applied Optics $23(6): 928-933$.

Molen, G. M. 1978. "Multiple Beam Interferometry." IEEE Transactions on Instrumentation and Measurement 27(3):246-249. 
Schwar, M. J. R., and J. R. Bowen. 1972. "Measurements of the Local Velocity of Shock and Detonation Waves by Schlieren Interferometry of Doppler-Shifted Light." J. Phys. D: Applied Physics 5:1561-1575.

Holography:

Matulka, R. D., and D. J. Collins. 1971. "Determination of Three-Dimensional Density Fields from Holographic Interferograms." Jour. of Applied Physics. $42(3): 1109-1119$.

Trolinger, J. D. 1975. "Holographic Interferometry as a Diagnostic Tool for Reactive Flows." Combustion Science and Technology 13:229.

Tomography:

Santoro, R. M., et al. 1981. "Optical Tomography for Flow Field Diagnostics." Int. J. Heat Mass Transfer. 24:1139.

Sato, S. 1981. "Flame Temperature Measurements from Multi-Directional Holographic Interferograms Using Computed Tomography Technique." Based on work presented at the 19th Symposium on Combustion, Japan, p. 46 (in Japanese). 



\subsection{PARTICULATE MEASUREMENTS}

Particle size and number density measurements are among the most important potential applications of laser diagnostics. Particles formed by combustion (e.g., soot) may be measured, as well as fuel droplets, solid particles, or powcers. Size and loading information is of tremendous importance in practical combustion and engineering processes. Such measurements have commonly been ottained by nonoptical techniques, and by white-light optical techniques that require particle extraction before analysis. Such methods have considerable limitations because particle extraction disturbs flowfields and temperatures, and generally lacks time resolution capability. The methods are also uncertain for the measurement of very small particles or particles that tend to stick together (agglomerate). In-situ optical methods do require access to the test region, however, and no method can measure all particle sizes over the range extending from much smaller to much greater than the wavelength of light.

For in-situ measurements, and particularly for combustion systems, practical considerations of optical access limit the amount of data available for particulate diagnostics. Although the theory of the interaction of light with particles (Mie theory) is well established, it is based on several assumptions about the shape, properties and distribution of the particles that may often be unknown or untrue. Thus, neither complete measurements nor a universal theory is available. Given this hardship, in-situ measurements in combustion systems often must use simple features of the theory that are relativeiy insensitive to the unknown particle properties. Particle diagnostic development has been slow, and many techniques are only just beginning to emerge. For this reason, this chapter is constructed somewhat differently from the remainder of the report, in that each technique is only briefly discussed. First, however, the framework for all the measurement techniques is presented, followed by discussion of the Mie theory and its implications.

\subsection{OUTLINE OF MEASUREMENT METHODS}

The particle measurement methods to be discussed are classified in Table 4.1. Two major factors affect the choice of a measurement technique--the 
TABLE 4.1. Size Ranges of Particle-Sizing Techniques

$$
\text { Particle Size (microns) }
$$

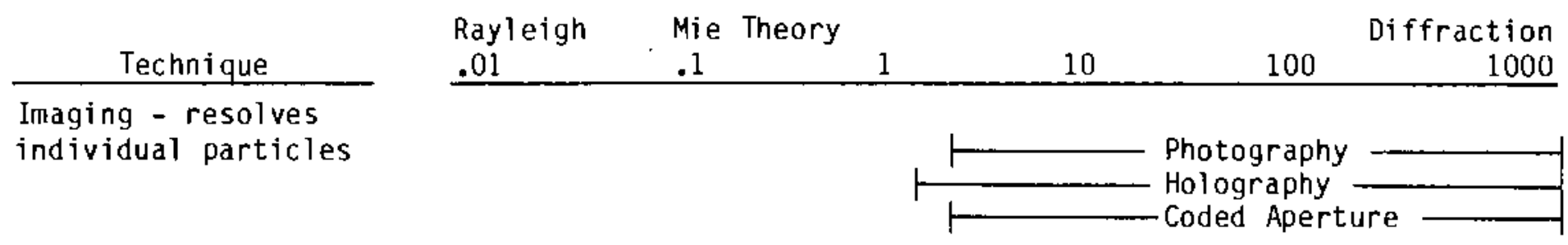

Light Scattering uses Mie theory

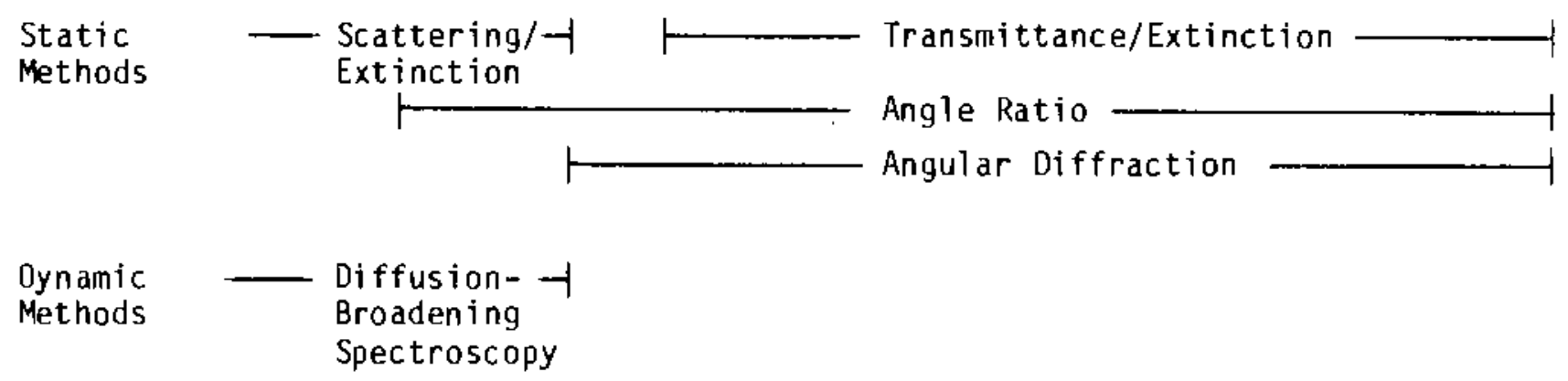

Single Particle Counters

Angle (and other) Ratios Absolute Intensity (deconvolution) Fringe Visibility (LDA) 
number density and the size of the particles to be investigated. For systems with very high number densities, no optical method will work, regardless of particle size. For less extreme systems, ensemble methods are possible; and finally, in relatively dilute systems, single particle-counting (SPC) lightscattering approaches are possible. Imaging methods vary in their ability to deal with high number densities--like the light-scattering (ensemble and SPC) systems, they do not work when there is multiple scattering of light from different test particles before the light is detected. Some methods have difficulties with depth of field as well.

Particle size, the second major technique determinant, dictates which simplifications to Mie theory are possible. Very small particles can use one simplification (Rayleigh); large ones can use another (forward diffraction). Imaging techniques are limited to relatively large particles. As imaging will not be covered in detail, it will be discussed next, before Mie theory and the range of light-scattering techniques are broached.

\subsection{IMAGING}

There are three major imaging methods: photography, holography, and coded aperture methods. Their resolution is set by the lenses they use. The high cost of diffraction-limited lenses, especialiy in larger sizes, effectively sets the practical lower size limit of the methods at 5 to 10 microns. In photography lasers can substitute for a strobe; several high-speed cameras and videos are available. Holography, which provides clear, three-dimensional pictures for which data reduction is very tedious, has been discussed in the previous section. The most recent coded aperture method estimates particie diameter from the ratio of the intensity of the image of the particle through two different slits, one bigger and one smaller than the particle. This technique can be effectively combined with particle temperature measurement (by pyrometry) in the investigation of burning coal particles, giving a powerful real-time measurement capability under the right conditions. 


\subsection{LIGHT SCATTERING AND MIE THEORY}

The intensity of scattered light depends on the shape and size of the particle or group of particles, on the number density, and on the index of refraction; it also varies with scattering angle and incident light wavelength and polarization. Finding particle size from measurements on scattered light, therefore, requires knowledge or assumptions about particle shape, size homogeneity, and index of refraction. Size measurements can involve finding scattering intensity as a function of angle or wavelength, or a variety of other schemes.

The most common relationship used to connect varticle size and light scattering data is Mie theory, which is the general mathematical theory of the electromagnetic scattering of light from homogeneous spherical particles with known complex refractive index. It is most accurate for single particle counting methods, because then the boundary conditions (including the input light) are well defined. The full Mie scattering solution is quite complex, and for large particles (greater than about 2 microns in visible light) it requires the summation of a great many terms of an infinite series. This is because the scattered light forms "lobes," as shown in Figure 4.1. The exact particle size limit derives from a size parameter which is proportional to the ratio of particle diameter over the light wavelength. The theory shows that for such particles, the forward (diffraction) scattering lobe predominates, which forms the basis for measurement simplifications. For very smail particles (less than 0.05-0.1 micron with visible light) the Mie solution can be closely approximated with a simple analytic result, which is the well-known Rayleigh scattering theory. The range between these extremes is from about 0.1 to 2 microns. In this range the full Mie theory is relatively tractable and the most accurate.

\subsection{ENSEMBLE MEASUREMENT METHODS}

\section{Small Particles}

Because the small particles of interest in combustion (basically soot) have relatively high number densities, only ensemble techniques can be used to measure them. Other types of small particles are of interest in various 


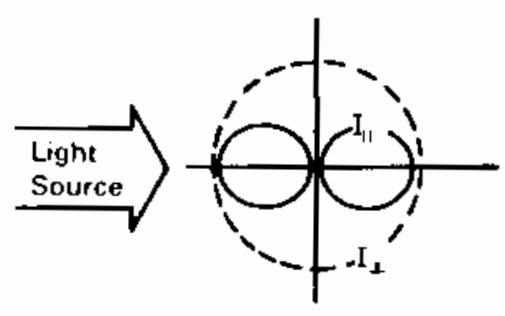

$$
a=\frac{\pi d}{\lambda}=0.1
$$

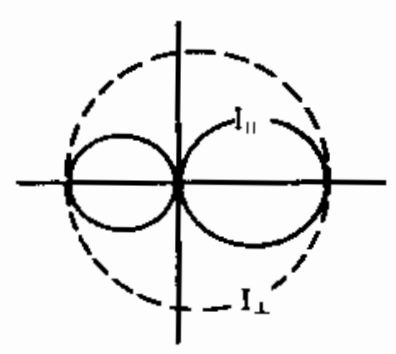

$a=0.8$

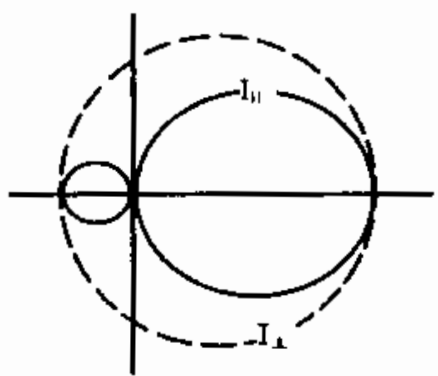

$a=1.6$

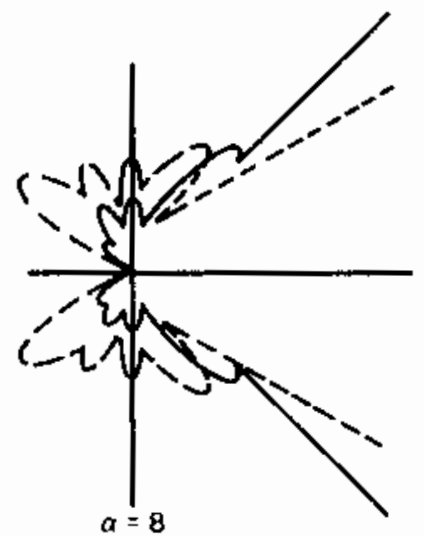

Increasing Particle Size

Diffraction Limit $a>10$

Rayleigh Limit

Intermediate

$l_{s}$ - Perpendicularly Polarized Light

$I_{31}$ - Parallel Polarized Light

d- Particle Diameter

$\lambda$ - Light Wavelength

Index of Refraction $m=1.25$ in Picture

FIGURE 4.1. Particle Light Scattering (From Mie Theory) 
applications as well. Methods discussed here are primarily ensemble techniques that are under development for soot measurement. These can be split into three classes, static light scattering (utilizing Mie and Rayleigh theory), dynamic light scattering (also called "diffusion-broadening spectroscopy"), and laser vaporization methods. The latter are not addressed here.

The Mie theory shows that a variety of scattering measurements can yield size information. These measurements include the intensity ratios at different angles, the ratio of perpendicular to parallel polarized scattered light, the extinction at different wavelengths, and the ratio of the scattered to extinguished light. For small particles where the Rayleigh limit applies, only the final measurement, called the scattering/extinction ratio, yields size (and number density) information. For larger particles the other measurements begin to be feasible. The same apparatus can be used for all the measurements when it makes sense to take them. The approach is simple, with good (but not threedimensional) spatial resolution. Signals are quite strong, so measurements are fast, little power is required, and equipment is relatively inexpensive. Disadvantages lie in the limitation of two-dimensional resolution and the sensitivity of the technique to refractive index, which is not well characterized for soot.

Another technique for small particles that is less sensitive to refractive index is dynamic iight scattering, also called diffusion-broadening spectroscopy or photon-correlation spectroscopy. The second name is most descriptive, as the technique is based on measuring the frequency shift from small particles undergoing Brownian motion. Brownian motion can be related to the particle diffusion coefficient, which in turn can be related to particle diameter when temperature and flow viscosity are known. This method gives good, threedimensional spatial resolution, is independent of refractive index, and is limited to smali particles (which have Brownian motion). Because of the disruptive effects of turbulent fluctuations, it is best used in low-velocity laminar flows. In addition, the electronics required to measure the frequency broadening are costly and complex, at least in comparison with a static scattering apparatus. 


\section{Large Particles}

The intensity distribution for the ensemble method for large particles is the superposition of many scattering lobes of different widths. Thus, particle size is "hidden," and various calculations (deconvolutions) must be made to extract it. In this sense, measurements are more difficult than for smaller particles under the Rayleigh limit. The scattering/extinction ratio outlined above is not very sensitive at larger particle sizes, but straight extinction measurements are possible. So are several of the ratios that were not effective for smaller sizes--angle, wavelength and polarization ratios. They are extremely sensitive to the value taken for the refractive index, however, and to nonsphericity. The polarization ratio can actually be used to measure nonsphericity. To minimize the sensitivity to refractive index, angle measurements can be made within the forward diffraction lobe, at least for larger particles where this lobe is large. A short discussion of each method follows.

Extinction measurements detect the transmission of the monochromatic laser beam through the dispersion of particles. If all the particles are the same size, the method will not provide number density and diameter separately--they are lumped together. If different sized particles are present with a fairly wide distribution, assumptions can be made that provide (given estimates of the particle volume or mass loading) an average diameter measurement that is relatively insensitive to refractive index. This method works for particles over one micron in size. Variants measuring the ratio of the transmissibility are also possible. Although this type of measurement does not provide point data, it can be used in extremely hostile environments. Measurements of ash droplets in magnetohydrodynamic (MHD) channels have been made by Self at Stanford, for instance. (a) Variants have also been applied to very small particles.

The various ratio methods first outlined for the discussion on small particles have all been tested for larger particles. The scattering/extinction ratio, which works well for the small particles, lacks sensitivity for larger sizes. Polarization measurements are difficult to make because small changes in the polarization lead to large changes in computed sizes. The sensitivity

(a) Dr. Sidney Self, Stanford University. 
to nonsphericity is also a factor--the depolarization ratio can be sensitive enough to be used as a sphericity indicator. Multiwavelength intensity measurements are not point measurements; hence, the technique is not as popular as the final technique, angle intensity ratio. It has been introduced primarily as a single particle counter, and will be discussed shortly. A variant for large particles, forward-angle diffraction, is an ensemble technique, however.

Forward-angle diffraction methods have been implemented in commercial particle-sizing instruments (see Figure 4.2). Because of their use of the forward scattering region, they are relatively insensitive to refractive index, though sensitivity does increase with particle size. They must employ a nonlinear deconvolution of some sort because the quantity they measure is a rather complicated ratio of moments of the particle size distribution function. This sets their accuracy limit. The size range limitations are set by characteristics of the scattered forward lobe as it relates to the collection aperture and the light beam to be scattered. The major disadvantage of the method, besides its inaccuracy, is that it offers no spatial resolution.

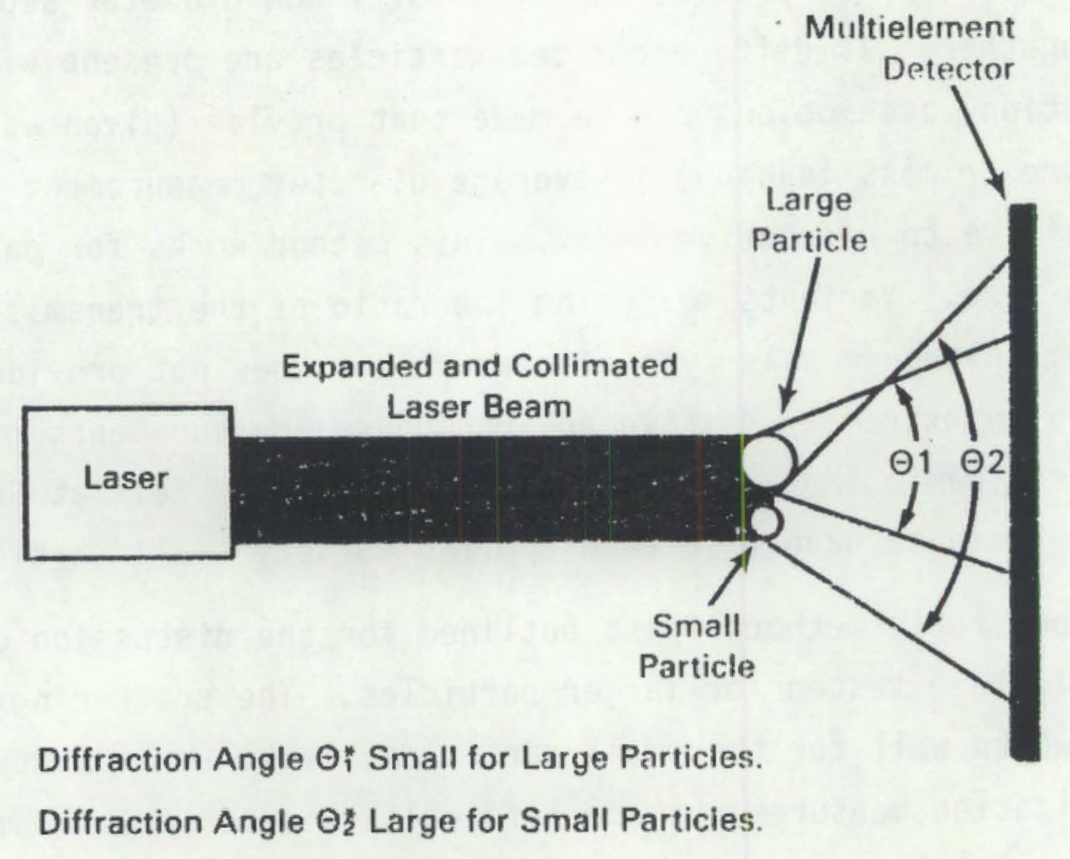

FIGURE 4.2. Forward-Angle Diffraction 


\subsection{SINGLE-PARTICLE COUNTERS}

Single-particle counters are for relatively disperse particle systems. In combustion, such systems consist of particles of intermediate size or larger; discussion here will thus focus on several methodologies for such particles. The first method, using the ratio of intensities measured at two different angles, has already been introduced. The second involves deconvolution of absolute intensity readings or absolute readings at small forward-scattering angles. These methods both use the configuration displayed in Figure 4.3. A third method utilizes laser Doppler anemometry (LDA) apparatus to make size measurements, and will be discussed in a separate section on LDA. In its basic implementation for particle work, this method involves a ratio as well: the visibility (amplitude-normalized scattering) ratio.

Angle ratio techniques can measure a wide range of particle sizes, as they are very sensitive to particle diameter. They can also give number densities. Their accuracy is limited by their sensitivity to index of refraction (which is why forward-diffraction methods were developed) and the effects of departures from Mie theory such as inhomogeneous or nonspherical particles. Accuracy, then, is not a strong point of the angle ratio method; it is perhaps best suited to use in the intermediate-size range where methods that avoid such problems do not work. When used for slightly larger particles only in the forward scattered light (as does the ensemble version), the accuracy improves,

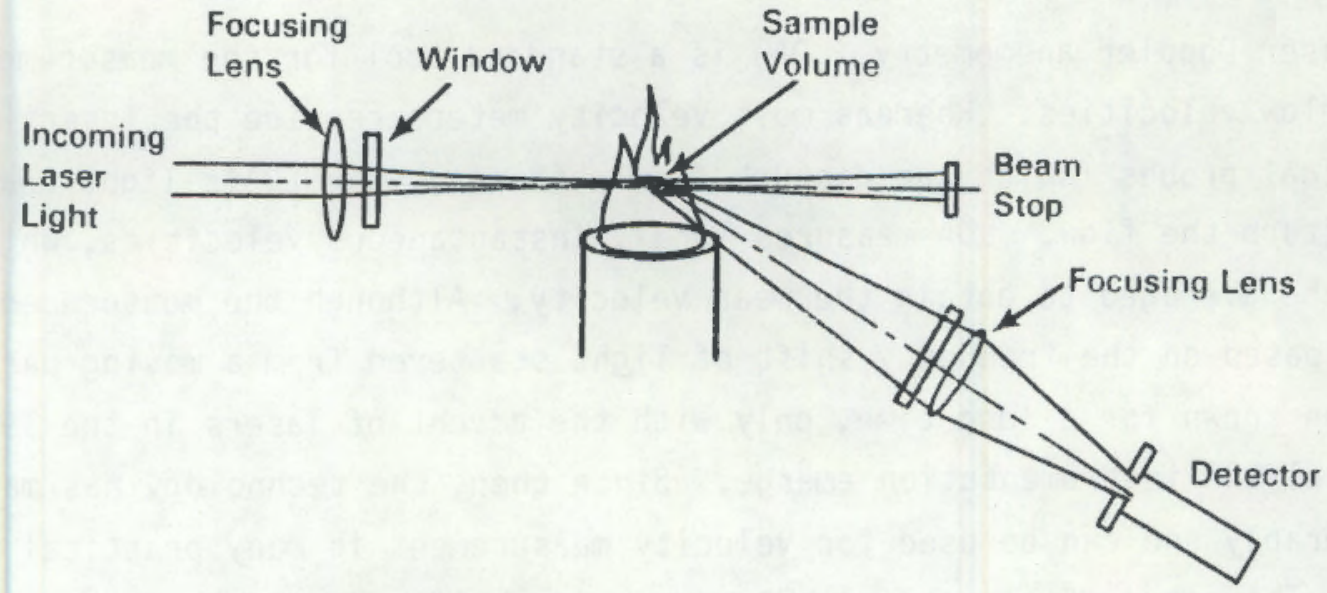

FIGURE 4.3. General Configuration for Single-Particle Counting 
with decreasing sensitivity to refractive index and sphericity. The angle ratio method is likely to err for broad size distributions, but measurements at multiple scattering angles can pinpoint misleading results because they are a function of local maxima from different scattering lobes. One factor hindering application of this method is the relatively complex equipment associated with it.

The absolute-intensity (via deconvolution) method is much simpler in its equipment, involving very sophisticated analysis instead. Single particle counters must always account for nonuniformities in the sample volume formed by the laser light. For the method above, the ratio provides this service but allows some error as well. For the LDA methods, nonuniformities become a source of significant error. The absolute-intensity method uses the nearforward scattering region because of its insensitivity to particle shape and refractive index. The system is calibrated with particles of known size and kept precisely aligned so that the intensity in the measurement volume does not change. Then, inversion algorithms accounting for various trajectories through the varying brightness of portions of the measurement volume can be used to give particle size distributions purely as a function of scattered light intensity. The method, though intuitively difficult, provides good spatial resolution and requires relatively simple equipment.

\subsection{LASER DOPPLER ANEMOMETRY}

Laser Doppler anemometry (LDA) is a standard tool for the measurement of local flow velocities. Whereas most velocity meters require the insertion of mechanical probes (which can disturb flow patterns), the LDA's light beams do not perturb the flow. LDA measures local, instantaneous velocities, which can easily be averaged to obtain the mean velocity. Although the measurement principle, based on the frequency shift of light scattered from a moving particle, has been known for a long time, only with the advent of lasers in the 1960s did practical LDA instrumentation emerge. Since then, the technology has matured considerably and can be used for velocity measurement in many practical situations. Thousands of reports on LDA have been published, and it has become increasingly sophisticated and a field of investigation in its own right. Some 
of the papers examine the use of LDA for particle sizing, as the value of simultaneous velocity and size measurements is considerable. Unfortunately, such efforts have proved to be difficult. The subject will be briefly discussed at the end of this section.

Background

Various optical configurations have been used for LDA measurements, in keeping with an increasing variety of applications. They are all based on the same measurement principle, however. In a typical application (Figure 4.4), two separate beams split from the same laser source are focused on a small element in the flow. Particles passing through this illuminated element scatter the light, which is then collected by a sensor. The signal is a modulation (change in frequency) of the incident laser light that is dependent on the particle's speed. It is often interpreted in terms of a fringe model, involving interference fringes set up in the measurement volume by the two lasers (see Figure 4.5) and their interaction with the particle. This model is not correct, however, as becomes evident when attempting to make particle size measurements. In that case the signal amplitude is related in a complex way to the particle's size, refractive index and trajectory. The velocity modulation

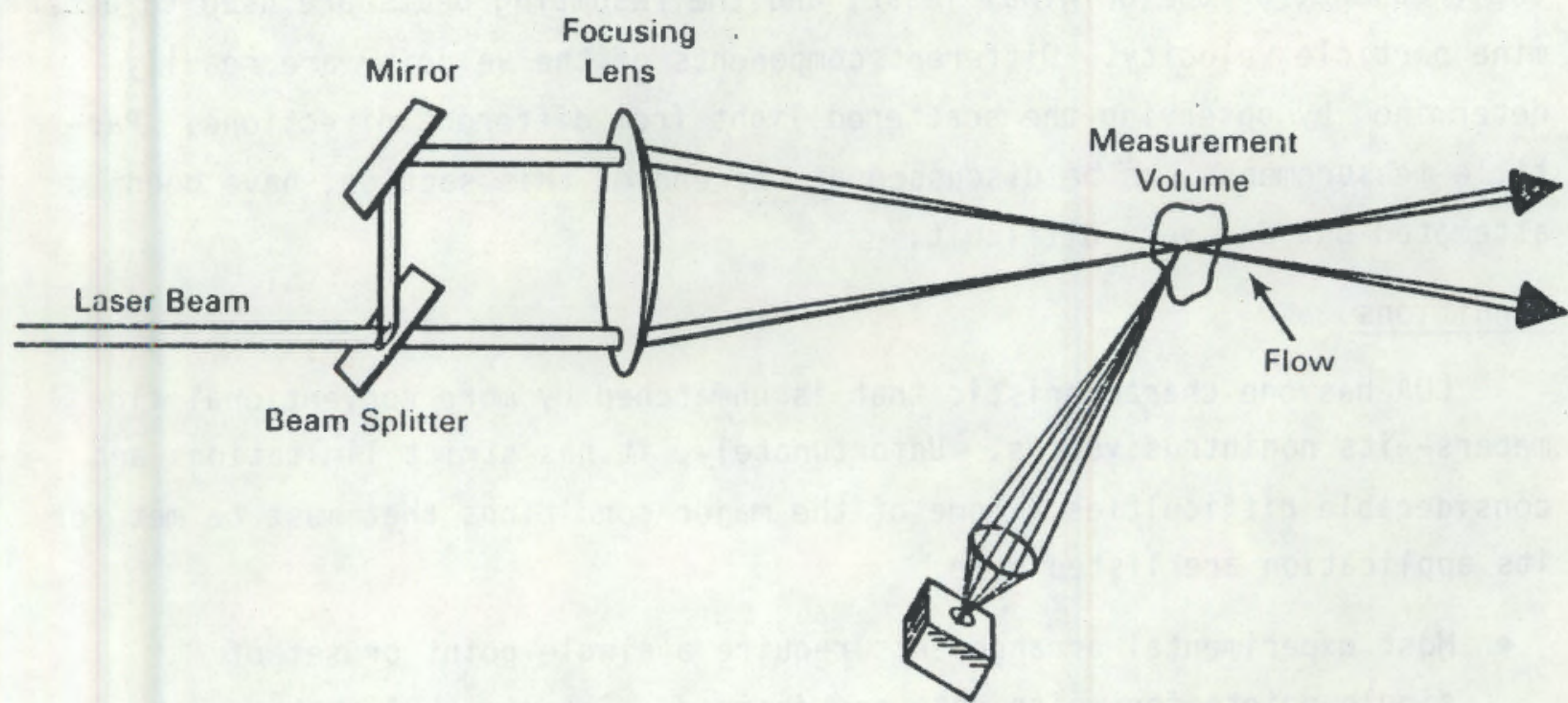

Pliotodetector

FIGURE 4.4. Basic Arrangement for Laser Doppler Anemometry 


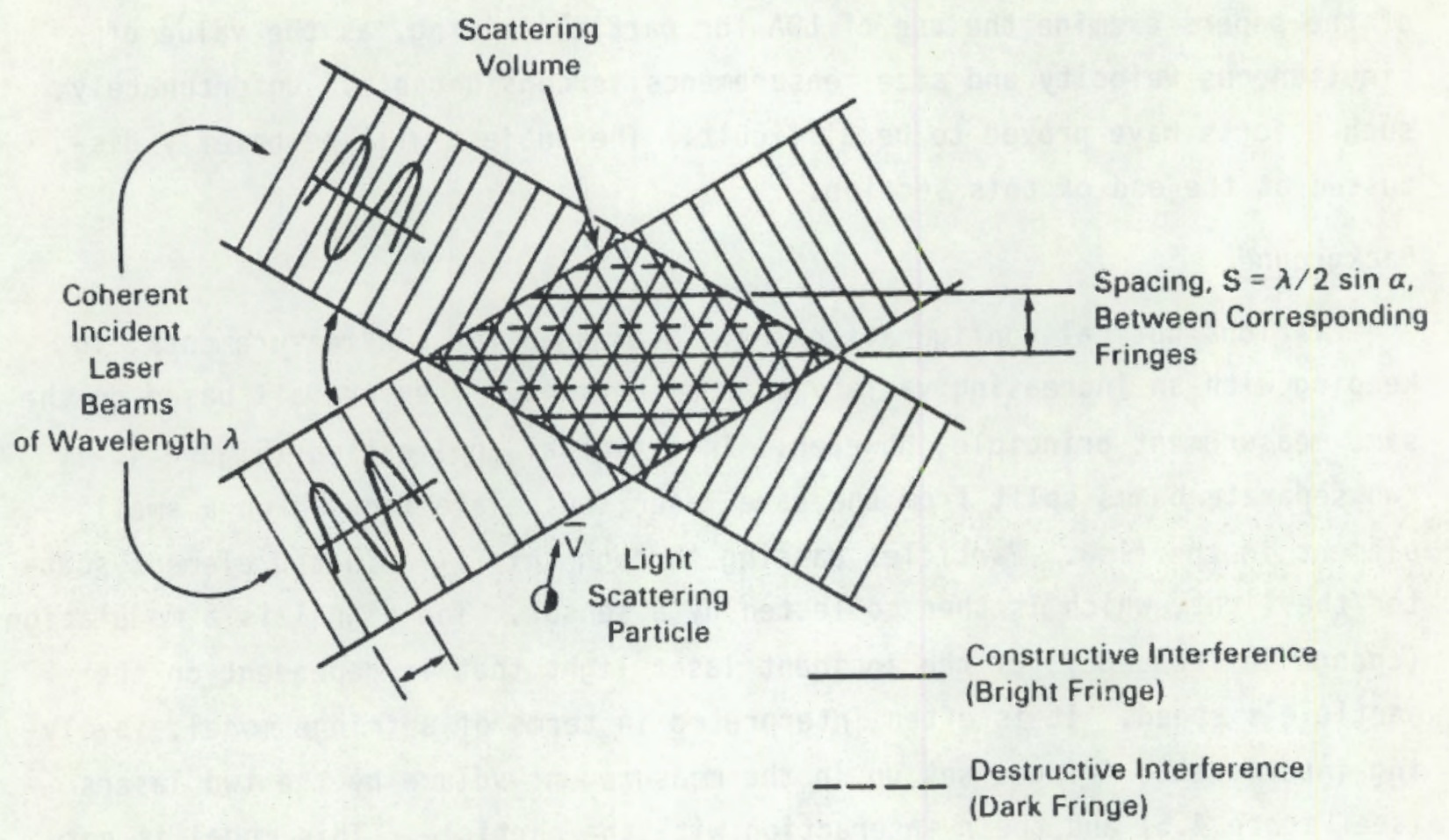

FIGURE 4.5. Fringe Interpretation of LDA Measurements

frequency is very high and difficult to measure, so it is usually mixed with the frequency of the original laser, and the resulting beats are used to determine particle velocity. Different components of the velocity are readily determined by observing the scattered light from different directions. Particle measurements, to be discussed at the end of this section, have been attempted but are very difficult.

\section{Conditions}

LDA has one characteristic that is unmatched by more conventional flow meters--its nonintrusiveness. Unfortunately, it has strict limitations and considerable difficulties. Some of the major conditions that must be met for its application are listed here:

- Most experimental arrangements require a single point or set of single points for which data are desired. Scanning LDA systems have been built for atmospheric mapping from airplanes, however. 
- The point(s) of investigation must be accessible by a light path from more than one direction, especially when several velocity components will be measured. This is difficult in rotating machinery and other intricate flow situations, or with opaque flows of various types.

- If there are no suitable light-scattering particles in the flow, some must be introduced. They must be small and have a much higher frequency response than the detector in order to appear to follow flow stream lines faithfully in sharp bends and velocity gradients. When turbulence is being measured, the particles should be especially small. Distribution must be carefully controlled, in a manner optimized relative to the detection electronics used. Finaily, the refractive index of the particle must be at least slightly different from that of the fluid.

\section{Major Difficulties}

Although LDA is now commonly used in research laboratories, the complexities of the method can limit its practicality. One obvious difficulty is alignment in configurations where two beams are used and must be independently focused on a small volume element. Changing the location of the measurement volume can aiso be difficult. Such problems, which are common to most laboratory LDA systems, can be solved with additional equipment, but the vibrations of systems under test may aggravate the situation. A second concern is particle distribution, for a sufficiency of particles must be relatively evenly introduced. Once in the flow, considerable--yet unquantifiabie--errors can be caused when particles do not follow streamines exactly. It is occasionally difficult to find acceptable particle sizes and materials for some flows.

Once the scattered 1 ight from the particles is obtained, there are further difficulties. A wide variety of signal-processing methods have been necessary to deal with the high frequency (and occasional weakness) of the primary signal. These can require sophisticated, expensive electronics. Often the complexity is also due to the need to average many signais (because of turbulence 
in practical flows) in order to obtain a statistically significant measurement. Some readings can take several minutes, although other transients, including certain turbulent flows, can be effectively "frozen". Thus, although LDA is perhaps the ideal tool for detailed probing of turbulence in properly structured experiments, its use in probing actual engineering flows must be carefully considered.

Current Work

The examples of current work described here are attempts to show several types of situations in which LDA measurements are advantageous. Because LDA technology is widely distributed and is even marketed as a system, this list is not meant to be complete. The most clear-cut case for use of LDA has been made for combustion flows. Their heat makes the use of mechanical probes impossible unless they are artificially cooled--which significantly perturbs the combustion environment. With proper filtration, even highly luminous flows can be probed by the LDA process. Other flows that are easily perturbed include separated flows, variable-property flows, and highly turbulent flows. LDA is very useful in measuring all of these types. Measurements are aiso often made in liquid flows, in low-velocity flows, and in rotating machinery. Systems that are more robust can and should be examined with simpler methods.

Particle Measurement

Many attempts have been made to extract particle size and number density information from LDA signals. As noted earlier, however, the amplitude and modulation depth of the signals depend on the size of the scattering particles, the optical properties of the particle material, the laser wavelength, the angle between the two incident laser beams, and the size and location of the sensor. Mie theory can be used to predict the amplitude of the LDA signal and its dependence on these parameters, but simplified models that are suitable to velocity measurements (e.g., the fringe model) have limited validity when applied to particles. Thus, "fringe visibility" particle measurements and other procedures have not been very successful in realistic situations. Limitations include sensitivity to scattering particle properties, strong dependence of the signal on scattering angle and on the collection optics geometry, and a narrow dynamic range. Signal validation is also very complex and causes 
difficulties. Recent work in Germany using white light sources and careful optical design based on the predictions of the full Mie theory may have more potential (Durst 1982); such methods can still be combined with LOA velocity measurements.

\subsection{REFERENCE}

Durst, F. 1982. "Combined Measurements of Particle Velocities, Size Distributions and Concentrations." Jour. of Fluids Engineering 104:284-296.

\subsection{BIBLIOGRAPHY}

Particle Measurement:

Bohren and Hoffman. 1983. "Absorption and Scattering of Light by Small Particles." John Wiley and Sons, New York.

Holve, D. U., et ai. 1981. "Design Criteria and Recent Developments of Optical Single Particle Counters for Fossil Fuel Systems." Optical Engineering $20(4): 529-539$.

\section{Imaging:}

Chigier, N. A., and C. G. McCreath. 1974. "Combustion of Droplets in Sprays." Acta Astronautica 1:687.

Grabowski, W. 1983. "Measurement of the Size and Position of Aerosol Droplets Using Holography." Optics and Laser Technology 199-201.

Jones, A. R., et al. 1978. "Application of In-Line Holography to Drop Size Measurement in Dense Fuel Sprays." Applied Optics 17:328.

Trolinger, J. D. and M. P. Heap. 1979. "Coal Particle Combustion Studied by Holog:aphy." Applied Optics 18:1757.

Tichenor, D. A., et a1. 1984. "Simultaneous In-Situ Measurement of the Size, Temperature, and Velocity of Particles in a Combustion Environment."

SAND 34-8628, Sandia National Laboratories, Livermore, California.

Small Particle Ensemble Methods:

Bonczyk, P. A. 1979. "Measurement of Particie Size by In-Situ Laser-0ptical Methods: A Critical Evaluation Applied to Fuel Pyrolyzed Carbon." Combustion and Flame 35:191-206. 
Driscol1, J. F., D. M. Mann and W. K. McGregor. 1979. "Subricron Particle Size Measurements in an Acetylene-0xygen Flame." Combustion Science and Technology 20:41.

Kadota, T., and M. Hiroyasu. 1984. "Soot Concentration Measurement in a Fuel Droplet Flame Via Laser Light Scattering." Combustion and Flame 55:195-201.

Menna, P., and A. D'Alessio. 1982. "Light Scattering and Extinction Coefficients in the Wavelength Range from $200 \mathrm{~mm}$ to $600 \mathrm{mrn} . "$ Nineteenth Symposium (International) on Combustion. The Combustion Institute, Pittsburgh, Pennsylvania, pp. 1421-1428.

\section{Large Particle Ensembie Methods:}

Caroon, T. A. and G. Borman. 1979. "Comments on Utilizing the Fraunhofer Diffraction Method for Droplet Size Distribution Measurements." Combustion Science and Technology 19:255.

Faxvog, F. R. 1978. "New Laser Particle Sizing Instrument." SAE Paper 770140, Society of Automotive Engineers, Warrendale, Pennsylvania.

Hirleman, E. D. 1982. "Calibration Studies of Laser Diffraction Droplet Sizing Instruments." Paper WSSI CI 82-54, Western States Section of the Combustion Institute, Pittsburgh, Pennsylvania.

Markham, D. L. 1981. "The Dispersion of Drop Sizes in Gas Turbine Fuel Nozzle Sprays." In Fluid Mechanics of Combustion Systems. ASME Special Publication, American Society of Mechanical Engineers, New York, New York.

Swithenbank, J., et al. 1976. A Laser Diagnostic Technique for the Measurement of Droplet and Particle Size Distribution. AIAA Paper, 76-79, American Institute of Aeronautics and Astronautics, New York, New York.

\section{Single Particle Counters:}

Hirleman, E. D. 1980. "Laser-Based Single Particle Counters for In-Situ Particulate Diagnostics." Optical Engineering 19:854.

Hirleman, E. D., and S. L. K. Wittig. 1977. "In-Situ Optical Measurement of Automobile Exhaust Gas Particulate Size Distributions: Regular Fuel and Methanol Mixtures." In Sixteenth Symposium (International) on Combustion, pp. 245-254. The Combustion Institute, Pittsburgh, Pennsyivania.

Hoive, D. J. 1983. "A Single Particle Counting Diagnostic System for Measuring Fine Particulates at High Number Densities in Research and Industrial Applications." SAND 83-8246, Sandia National Laboratories, Livermore, California. 
Holve, D. J., et ai. 1981. "Design Criteria and Recent Developments of Optical Single Particle Counters for Fossil Fuel Systems." Optical Engineering 20:529.

LDA:

Blint, R. J., and J. H. Bechtel. 1982. "Flame-Wall Interface: Theory and Experiment." Combustion Science and Technology 27:87.

Buchhave, P., et a1. 1979. "The Measurement of Turbulence with the LaserDoppler Anemometer." Annual Review of Fluid Mechanics 11:443-503.

Durano, D. F. G., et al. (eds). 1982. Applications of Laser Doppler Anemometry to Fluid Mechanics. (Proceedings) International Symposium, July 5-7, 1982. Lisbon, Portugal.

Durst, F., A. Melling and J. H. Whitelaw. 1976. Principles and Practice of Laser-Doppler Anemometry. Academic Press, New York.

Goldstein, R. J., K. Y. Lau and C. C. Leung. 1983. "Velocity and Turbulence Measurements in Combustion Systems." Experiments in Fluids 1:93-99.

Johnston, S. C., et al. 1979. "Application of Laser Diagnostics to an Injected Engine." SAE Paper 790092, Society of Automotive Engineers, Warrendale, Pennsylvania.

Kennedy, I. M., and J. H. Kent. 1981. "Solar Measurements in a Co-Flowing Turbulent Diffusion Flame." Combustion Science and Technology 25:109.

Mizushina, T., et al. 1978. "Application of Laser Doppler Velocimetry to Turbulence Measurement in Non-Isothermal Flow." Proc. R Soc. 366:63-79. London, England.

Thompson, H. D., and W. H. Stevenson, eds. 1978. Laser Velocimetry and Particle Sizing. Hemisphere Publishing Company, Washington, D.C.

Witze, P. 0. 1980. "A Critical Comparison of Hot Wire Anemometry and Laser Doppler Velocimetry for I. C. Engine Applications." SAE Paper 800132, Society of Automotive Engineers, Warrendale, Pennsylvania.

Yoshida, A., and H. Tsuji. 1979. "Measurement of Fluctuating Temperature and Velocity in a Turbulent Pre-Mixed Flarne." Seventeenth Symposium (International) on Combustion, pp. 945-955. The Combustion Institute, Pittsburgh, Pennsylvania.

\section{LDA for Particles:}

Adrian, R. J., and K. L. Orloff. 1977. "Laser Anemometry Signals: Visibility Characteristics and Applications to Particle Sizing." Applied Optics 16:677. 
Farmer, W. M. 1972. "Measurement of Particle Size, Number Density, and Velocity Using a Laser Interferometer." Applied Optics 11:2603-2612.

Maeda, M., et al. 1982. "Velocity Measurements of Dispersed and Continuous Phase in Suspension Flow by a Modified LDV System." Trans. of the JSME. Ser. B, pp. 48-425, 78.

Marko, K. A., and L. Rimai. 1982. "Single Particle Velocity and Trajectory Measurements of Microscale Inhomogeneities in Unstable Airflows." Optics Letters 7:130.

Reberds, D. W. 1977. "Particle Sizing Using Laser Interferometry." Applied Optics 16:1861. 


\subsection{SPECTROSCOPIC DIAGNOSTICS}

Spectroscopy has long been the province of physicists and chemists seeking the information it provides about the structure of matter. As knowiedge accumulated, spectroscopy developed into an analytical tool for identifying substances. Most of this work was done with solids and liquids, as only condensed phases provided adequate signals. With the advent of lasers, however, work with gases expanded, and spectroscopy evolved into an engineering tool to be used for solving problems. Several scattering processes are used in this fashion. Those that have been developed into useful diagnostics are examined in this chapter.

As noted above, spectroscopic methods provide information about the structure of the molecule being probed. If that structure (i.e., the spectrum) is known, these techniques can be used to probe for and quantify concentrations and temperature of a species. All the light scattering on this molecular level is much weaker than the particle scattering outlined above. Rayleigh scattering, asplicable only to the very smallest of particles, is aiso applicable to molecules-it is the elastic scattering process (same energy and nence same frequency). It can provide direct density and temperature measurements. Laser-induced fluorescence is a strong scattering process best suited for measuring 10 w concentrations of simple molecules, such as chemical intermediates in flames, since despite its sensitivity it is unusable in dirty or pressurized environments for which there is excessive unwanted fluorescence. The most versatile inelastic method in common use is spontaneous Raman spectroscopy. Although it is a very weak process, it is well understood and used in a variety of research fields. Finally, there are nonlinear light-scattering processes where scattering intensity is not proportional to input laser intensity. The major current example is coherent anti-Stokes Raman spectroscopy (CARS), a light-mixing process that provides similar information to ordinary Raman but is usable (at least for temperature measurements) in practical (e.g., bright and dirty) environments. Other nonlinear processes are under investigation as well. These and other new approaches may add capabilities to those of the present primary tools. Some newer approaches are discussed briefiy at the end of the sections on related techniques or at the end of this chapter. 


\subsection{LASER - INDUCED FLUORESCENCE}

Laser-induced fluorescence (LIF) is a diagnostic technique capable of measuring gaseous species in extremely low concentrations. It is most suitable for detecting certain reactive intermediates such as unstable flame radicals. In addition to being nonintrusive and remote, it can provide good spatial and temporal resolution. LIF has significant advantages over older methods used for flame radical measurements before the advent of tunable lasers (absorption or emission spectroscopy). Emission spectroscopy only provides information about the excited state of a species, not (as does fluorescence) the much larger and more chemically significant population of the species in the ground state. Absorption spectroscopy gives ground-state information but provides line-of-sight measurements only--the data are effectively integrated along the probe beam and are not spatially resolved. Thus, fluorescence is an important tool for unraveling the complex processes controlling combustion efficiencies and pollutant formation, plasma chemistry, and other chemical reactions. LIF variants can be used to measure temperature and, in certain cases, velocity as well. Work is also progressing on two-dimensional LIF using a sheet of laser light and an array of detectors. This technique is especially useful for imaging complex reacting flows.

Background

Fluorescence is the spontaneous emission of radiation from an upper electronic state of an excited molecule. In LIF the excitation is caused by absorption of a photon of laser radiation tuned to coincide with a molecular resonance. The ensuing fluorescence may be at the resonant frequency or a different (usually lower) frequency if the molecule returns to a state different from the one in which it began. The nonresonant frequencies suffer less interference from elastic (Rayleigh) scattering and are therefore generally selected for viewing. Figure 5.1 outlines the basic fluorescent scheme. A variant of LIF uses two or more photons to excite the resonance state. It is useful for a number of species and is one of the best methods for detecting singlet atoms. 

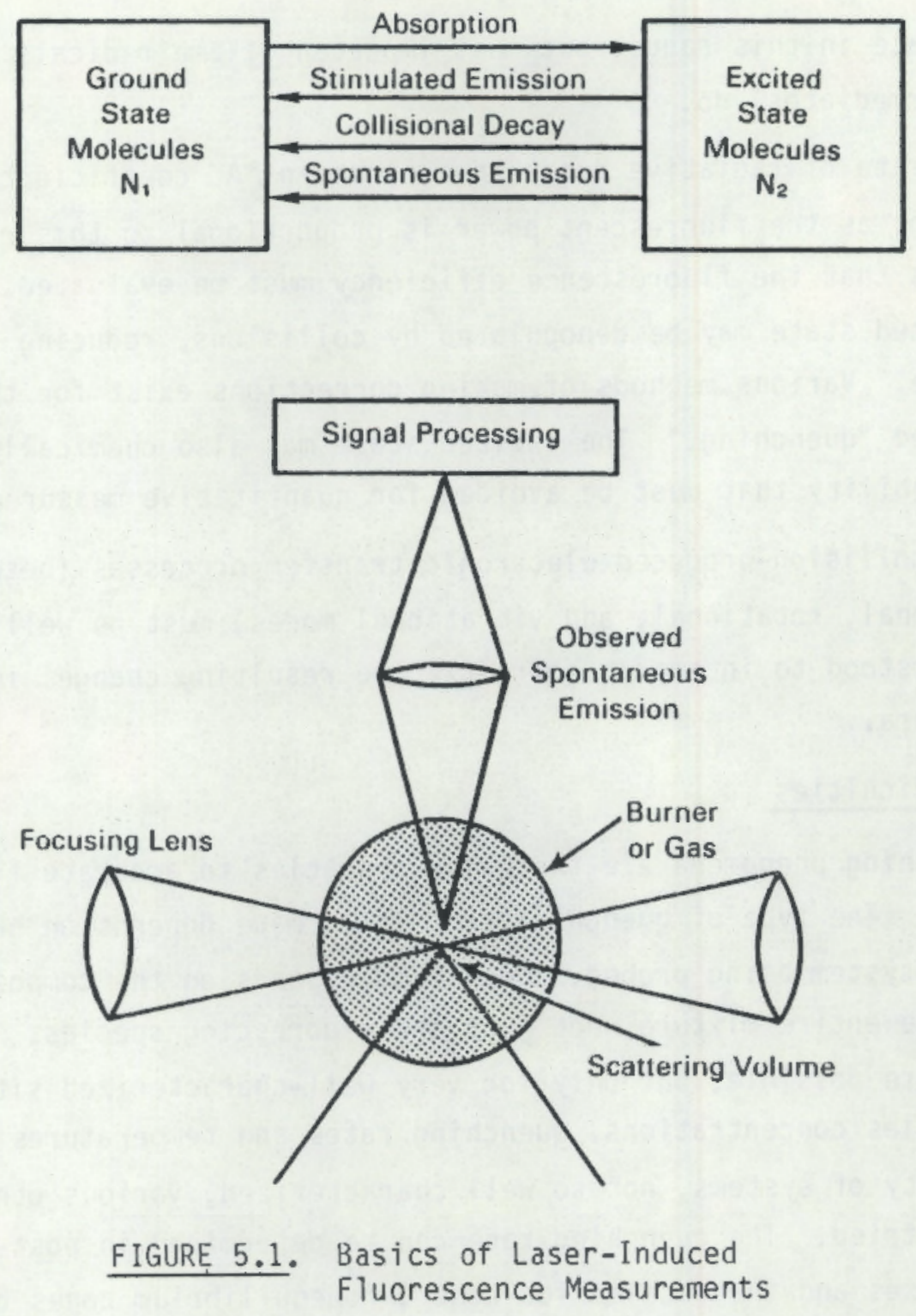

\section{Conditions}

In order to make fluorescence measurements on a given molecule, several basic criteria must be satisfied:

- The emission spectrum of the atom or molecule must be known; it must also not dissociate before emission.

- The atom or molecule must have an absorption wavelength that can be reached with tunable laser sources. The current range is approximately 2,000 to 15,000 angstroms. Many important molecules do not 
radiate in this range, but most important flame radicals (reactive intermediates) do.

ه The rate of radiative decay (the Einstein "A" coefficient) must be known, as the fluorescent power is proportional to this rate. This means that the fluorescence efficiency must be evaluated. The excited state may be depopulated by collisions, reducing the fluorescence. Various methods of making corrections exist for this process, called "quenching." The excited state may also chemically react--a possibility that must be avoided for quantitative measurements.

- The collision-produced electronic transfer processes (between translational, rotational, and vibrational modes) must be well enough understood to interpret correctly the resulting changes in the LIF spectra.

Major Difficulties

Quenching phenomena are the major obstacles to accurate fluorescence measurements. The type of quenching correction made depends on how much is known about the system being probed. Quenching depends on the composition and pressure of the entire mixture, not just the fluorescing species. Analytical corrections are possible, but only for very well-characterized situations where major species concentrations, quenching rates and temperatures are known. For the majority of systems, not so well characterized, various other approaches have been tried. The quenching rate can be determined in post-reaction equilibrium gases and then scaled for other, nonequilibrium zones by using kinetic collision theory. Fluorescence decay following a laser pulse can be used to deduce the quenching rate, although the decay is very rapid and difficult to measure. For very short pulse times (《 quenching time), fluorescence collected during the pulse is independent of the quenching rate, although these measurements are also extremely difficult to make.

A final approach, saturation of the fluorescence signal, has received considerable attention. The incident laser intensity is made large enough that the absorption and stimulated emission rates are much larger than the quenching rate. Models of the rates of state population and depopulation have then been 
used, with some success, to analyze measurements. In addition, the resultant maximized signal also gives the best possible species detection sensitivity. The necessary high laser intensities are sometimes difficult to obtain, however; and unevenness in the longer laser pulses used reduces accuracy significantly. Such spikes in pulses can cause problems, with other measurement schemes as well. Finally, excited-state reactions or other forms of nonequilibrium behavior are more likely in saturation measurements, and can complicate data reduction.

There are other difficulties besides collisional effects in making LIF measurements. When relatively high concentrations of a molecule are examined, corrections must be made for the absorption (in transit) of the input laser and the scattered signal. The variances of laser lineshape can complicate this absorption calculation. A second difficulty is the determination of detector efficiency and the observed volume in the experiment, generally done by making a Rayleigh or absorption measurement on a gas of known concentration in the experimental rig. A similar approach is used for much of the most recent LIF work, which has concentrated on relative rather than absolute measurements. When absolute numbers are needed, an absorption or Raman measurement is made to provide a baseline value.

A great deal of work is required to make a LIF measurement in most flames or mixtures. Crosley and Smith (1983) note the tremendous need for active consultation between spectroscopists doing fundamental work and qualified users interested in LIF applications. More work is needed on excited-state lifetimes and transfer mechanisms, transition probabilities, and collision phenomena of almost every species of possible interest. If such work is completed for those of greatest importance, it will help in determining feasible detection strategies and in making new and more accurate measurements. Even with such information, making any but the most basic of measurements requires a great deal of competence in spectroscopy as well as chemistry.

Current Work

Limited spectroscopic data have directed most LIF work toward better-known or particularly interesting species. Spectroscopic work and occasional applications research have been done with atoms, molecules and flame radicals. 
Sodium (Na) has received considerable attention because of its energy level simplicity and accessible absorption characteristics, as well as its frequent use as a tracer. Other atoms examined include lithium, zirconium, tantalum, indium, iodine, and metals such as copper, magnesium, and chromium. Probably the most-studied molecular species is $\mathrm{NO}_{2}$, because of its broad absorption spectrum in visible light. Other molecules of interest have included NO, CO, $\mathrm{N}_{2}, \mathrm{O}_{2}$, and sulfur compounds. Radicals under study have included $\mathrm{OH}, \mathrm{CH}, \mathrm{NH}$, and many others. $\mathrm{OH}$, a very important radical in hydrocarbon/air flames and pollutant formation processes, has received by far the most attention. This popularity is due partly to its importance, and partly to a considerable spectroscopic data base from basic research performed much earlier. Another advantage relates to the fortuitous frequencies at which strong $\mathrm{OH}$ transitions are located that result in their being easily and repeatably reached by tunable lasers. Results of the flame radical work have been used in chemical kinetics modeling, and sulfur studies have provided useful insights into sulfur chemistry, with a possible bearing on acid rain.

Temperature and Velocity

Fluorescence can be used for temperature and velocity measurements as well as species determination. There are several common approaches for temperature measurements. In two-line fluorescence, temperature is determined from the ratio of intensities of two transitions originating at the same upper state. In another approach, the temperature-dependent population of a seed atom's upper electronic state is measured, in many cases from its rotational branch spectrum, and then the temperature is calculated. Seeding the flow with an unreactive species greatly extends the temperature range of LIF. At low densities and pressures, as in hypersonic flow, temperature broadens the resonance fluorescence line width in a given rotational or vibrational state. Fortunately, this effect is to some extent predictable. The problems related to temperature measurement generally arise from the same causes as species measurement: quenching, excited-state transitions, and scattering and laser intensity variations and the difficulties of alleviating or accounting for them. These problems currently create significant uncertainty in most LIF temperature measurements. 
Velocity measurement using LIF is not a widespread technique. This method and several others involving various spectroscopies measure the velocities of actual atoms or molecules in the flow and thus do not (as does LDA) suffer the inaccuracies inherent in measuring particles carried by a flow. With LIF, velocity measurement involves determining the Doppler shift of absorption by a known atomic or molecular species, as excited by a single-mode (single-frequency) laser. The known species, preferably one that is readily excited yet does not disturb the flowfield, must generally be introduced. Some success has been had with sodium vapor, in high temperature flows, and iodine for ambient flows (McDaniel, Hiller and Hanson 1983). For supersonic velocities, the Doppler frequency shift is considerable and is fairly simple to measure accurately. Low velocities require somewhat more complex approaches in order to determine small frequency changes.

Planar LIF

Some of the most recent efforts in velocity measurements have utilized a new two-dimensional variant of LIF called planar LIF, or PLIF (Figure 5.2). It has also been used for simultaneous multipoint measurements of temperature and species concentration. The usual exciting laser beam is expanded into a thin sheet of light, and the resulting fluorescence is collected by an image-intensified camera at right angles to the sheet. The output can be stored digitally and processed to yield species, temperature or velocity distributions. This imaging and storing process introduces significant difficulties beyond the normal concerns of fluorescence measurement. For example, accuracy can become dependent on the noise characteristics of the photomultipliers used. The value of such images to modelers, in particular, however, appears to be immense.

\section{Multiphoton Fluorescence}

Multiphoton methods involve the use of two or more photons to excite the resonant state that fluoresces. This allows the excitation of species for which no suitable one-photon lasers exist or for which background gases absorb single photons. Multiphoton techniques are made possible by the availability of powerful, pulsed, tunable lasers. The fluorescent photons produced by this method generally have energies different from other possibly interfering photons, so that high sensitivities are permitted. The method has even been 


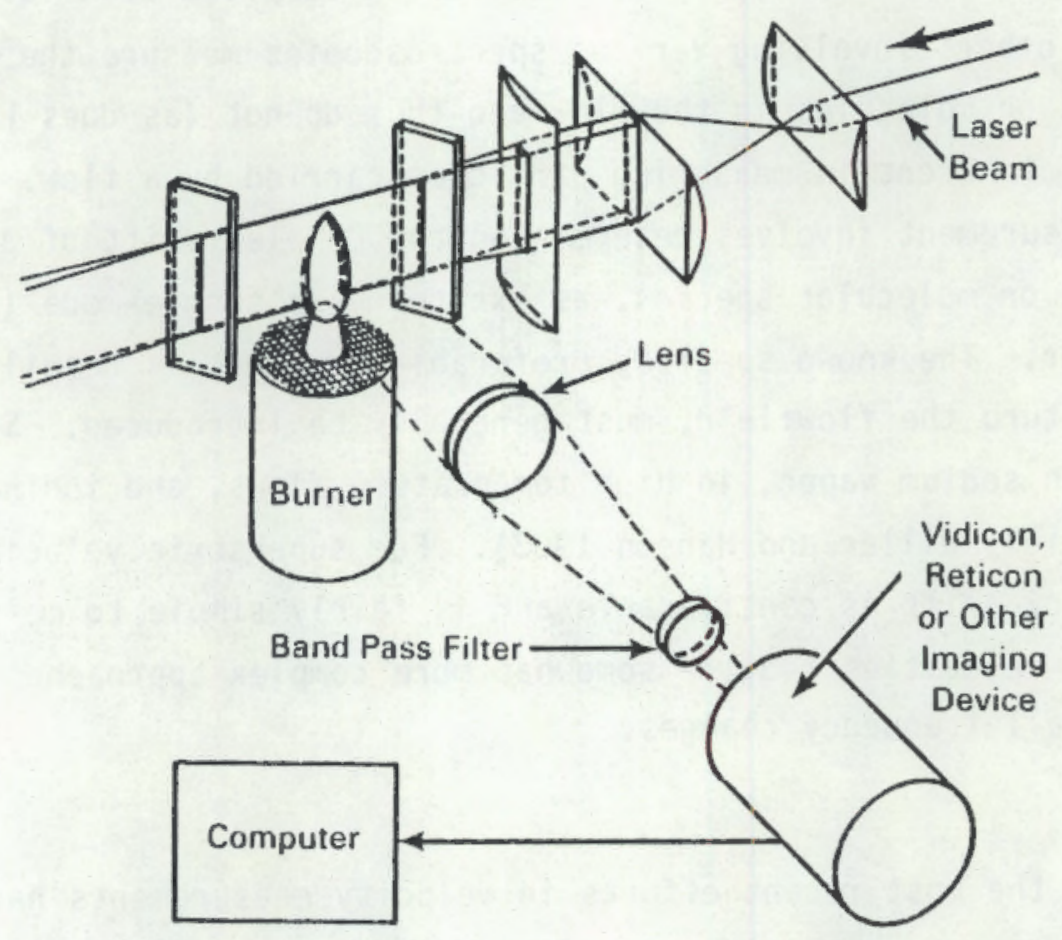

\section{FIGURE 5.2. Experimental Arrangement for Two-Dimensional} Laser Fluorescence Imaging

used to detect atomic species such as nitrogen and oxygen in flames. Accuracy is limited, however, because the process is nonlinear in laser intensity, and because two-photon cross-sections are not well known.

\subsection{SPONTANEOUS RAMAN SCATTERING}

Raman scattering, a process first observed in 1928, has been used by chemists for many years as an analytical tool for liquids and solids. With the advent of lasers, its promise as a combustion gas diagnostic became evident, and it has received considerable attention in the past 15 years. Spontaneous Raman, the basic form of the scattering, is perhaps the simplest, spatially precise, species-specific diagnostic available. The only requirement is a single, nontunable laser, generally operating in the visible region. With proper detection methods, the major species can all be measured at the same time with good space and time resolution. Since scattered-light intensities are proportional to the species density and are unaffected by collisional 
quenching, calibration is not difficult. In addition, the intensity is functionally dependent on temperature, and yields good temperature measurements. Unfortunately, spontaneous Raman scattering is weak, ten orders of magnitude less intense than fluorescence, for example, and hence can only be used in particle-free, nonluminous systems. Alternative, more complex scattering processes have been used to help circumvent this problem, and will be dealt with in a section on nonlinear techniques.

\section{Background}

Raman scattering is an inelastic collision process between photons of light and solid, liquid or gaseous molecules. In elastic collisions (called "Rayleigh scattering"), the photon has the same energy after the collision as before. In an inelastic Raman collision, the photon either has less or more energy. This shift to a new energy is dependent on the molecule involved in the collision by a precise amount. If the photon excites the molecule to a higher rotational or vibrational state, and loses energy itself, the shift is called "Stokes scattering." This is more likely than anti-Stokes scattering, where a molecule that happens to be excited before the collision gives up energy to the photon. Far fewer molecules are in excited states than are in the ground state. The intensities of the anti-Stokes lines are therefore less than those of the Stokes lines, which are in turn three to four orders of magnitude less than the Rayleigh line (which is at the laser frequency). The Stokes and anti-Stokes Raman lines are arrayed symmetrically about the Rayleigh line because the photon has either gained or lost the precise amount of energy involved in a particular transition. Figure 5.3 illustrates this arrangement.

The rotational transitions are about one order of magnitude stronger in intensity than the vibrational ones, but they are very close to each other and to the Rayleigh line. Therefore, the vibrational lines produce the most distinctive spectral pattern and are used for most Raman work. They, and the other transitions, occur because of the laser's frequency, not its intensity; since the signal is essentially a modulation of the input radiation, excitedstate quenching and other nonlinear effects are not significant. Raman signals are easily analyzed, therefore, since they are linear functions of the species 


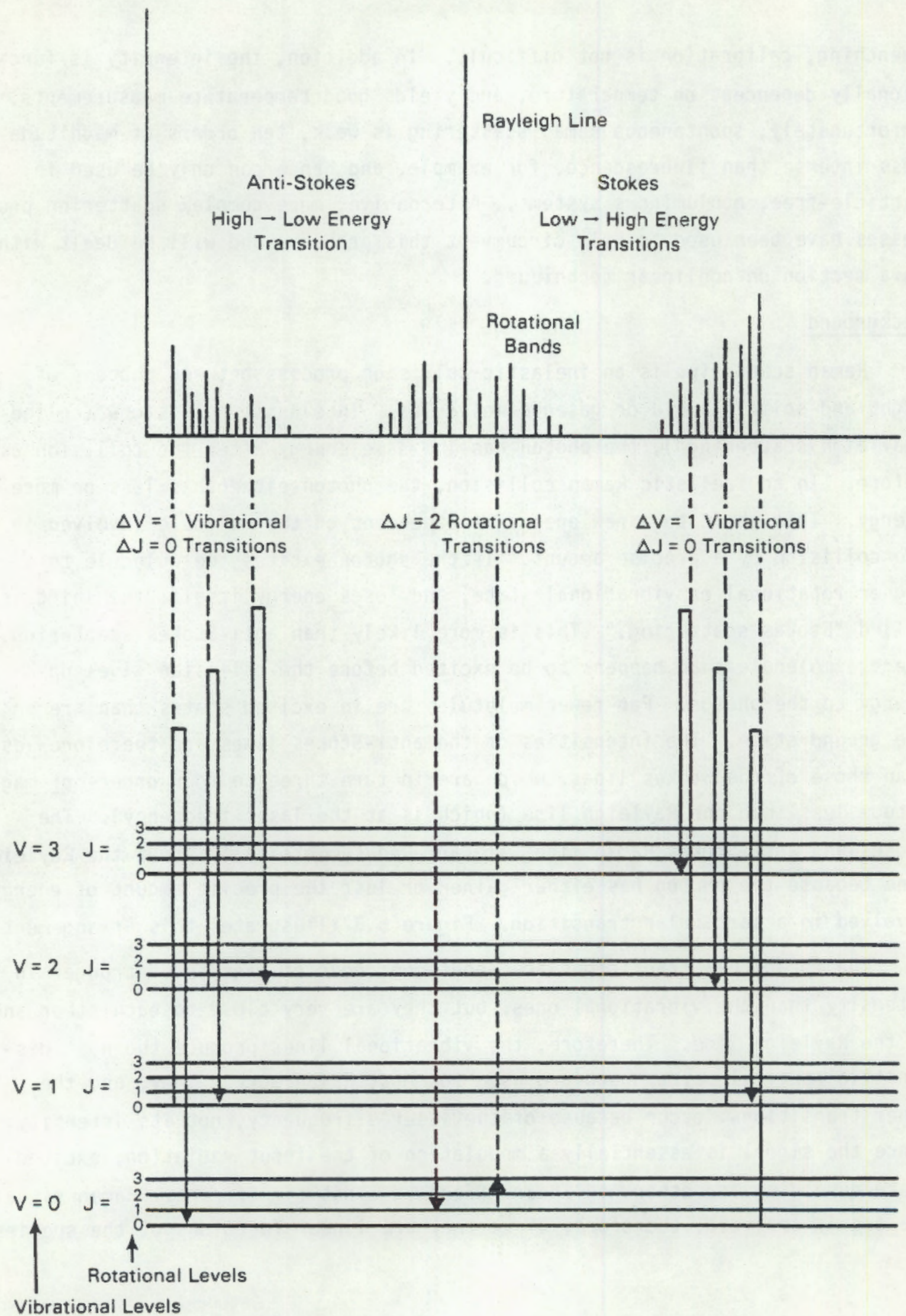

FIGURE 5.3. Outline of Basic Raman Transitions 5.10 
density and the laser intensity (this is only approximately true at high temperatures). Unfortunately, the relative intensity of the Raman scattering is three orders of magnitude less than Rayleigh scattering, ten orders of magnitude less than molecular fluorescence, and as much as 20 orders of magnitude less than Mie (particle) scattering. This limits its application considerably, to systems where the weak Raman signals can still be seen. These systems can be gas, liquid or solid phases. The arrangement for solid-phase experiments is shown in Figure 5.4 .

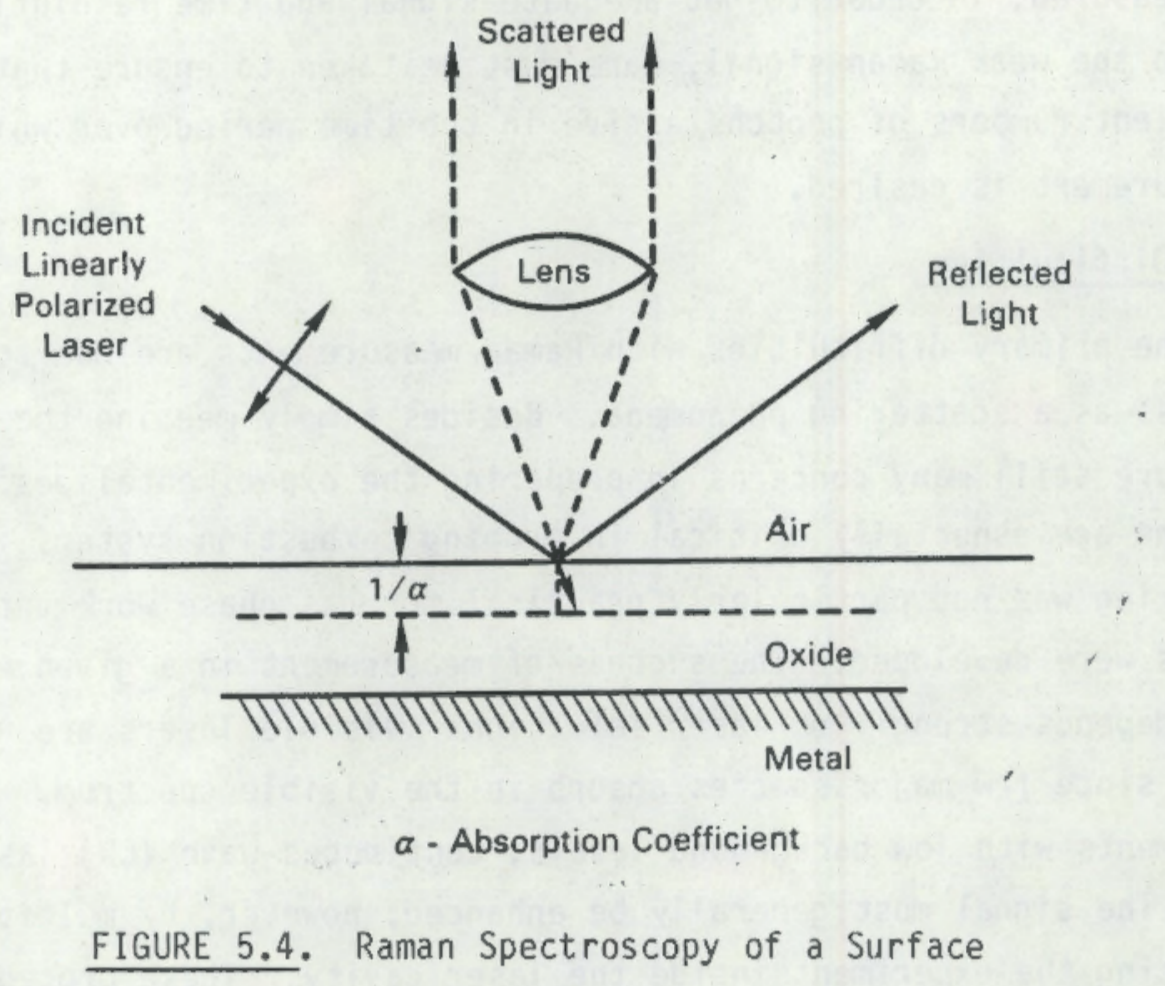

Conditions

To make spontaneous Raman measurements on a particular system, several basic criteria must be satisfied:

- Background radiation in the system must be low because the Raman signal is very weak. In a combustion system, this means that the flame luminosity, particle incandescence, fluorescence and Rayleigh scattering should be low. 
- The Raman spectral distributions of the species measured must be known. These are readily found from molecular transition cross-sections (probabilities) for many molecules at low temperatures, but at high temperatures they must be carefully calculated because of nonlinear effects.

- Only major species (> $1 \%$ mole fraction) measurements can be made.

- The measurement apparatus must be well matched to the system being measured, in order to get adequate signal and time resolution. Due to the weak Raman signal, care must be taken to ensure that sufficient numbers of photons arrive in the time period over which a measurement is desired.

Major Difficulties

The primary difficulties with Raman measurements are due to its relative weakness as a scattering phenomena. Besides simply meeting the criteria above, there are stili many concerns in preparing the experimental design. These concerns are especially critical in probing combustion systems. Indeed, Raman scattering was not particularly practical for gas phase work until laser light sources were developed. The success of measurement in a given environment still depends strongly on laser selection. Visible lasers are used in most cases, since few major species absorb in the visible spectrum. For simpler experiments with low background levels, continuous-wave (CW) lasers are suitable. The signal must generally be enhanced, however, by multipass methods or by placing the experiment inside the laser cavity. These procedures cause errors in finding time-averaged flow properties.

Pulsed lasers are used for "instantaneous" measurements or for probing luminous environments. With care, a pulsed laser can be chosen which yields a signal that can be detected above the background. If more than one pulse is necessary to accumulate enough photons for a statistically significant signal, time-averaging problems arise again. Once a suitable Raman signal is produced, it must be interpreted. Signals for different species can overlap, especially at high temperatures, and analysis can be complex. This can preclude measurements of some combinations of species, particularly of more complex, asymmetric 
molecules with complex spectra. Further difficulties associated with temperature measurement will be discussed shortly.

Current Work

A considerable amount of work is being done with spontaneous Raman scattering. Much of it is in laboratory combustion diagnostics, primarily work in clean laminar premixed $\mathrm{flames}$ and jets to detect major species such as $\mathrm{NO}_{2}, \mathrm{H}_{2}$, $\mathrm{CO}, \mathrm{CO}_{2}, \mathrm{H}_{2} \mathrm{O}, \mathrm{CH}_{4}, \mathrm{C}_{2} \mathrm{H}_{2}, \mathrm{C}_{3} \mathrm{H}_{8}$, and $\mathrm{NO}$. In many experiments, multiple species have been detected simultaneously. Application has also been made to practical devices, aithough test conditions generally had to be carefully selected. A variety of internal combustion (IC) engine research has been successful, aided by the increase of Raman signal intensity with pressure because of higher molecular densities. The flame/wall interface of a stable premixed flame has been examined (Bechtel and Blint 1980), increasing knowledge of the influence of cool interfaces on combustion. A final set of examples includes the numerous pulse laser Raman studies of turbulent flames, which have aided understanding of system chemistry and fluid dynamics.

\section{Temperature}

The Raman spectral distribution is essentially a display of weighted quantum state populations. Temperature measurements can therefore be performed from the shape of the distribution in a number of ways. Contour fitting to a known (theoretical) standard is perhaps the most accurate. The Stokes/antiStokes intensity ratio, peak height ratios, or bandpass ratios can also be used. The $\mathrm{N}_{2}$ lines are the best indicators in air-fed combustion systems, since $\mathrm{N}_{2}$ is then the dominant constituent regardless of the extent of the reaction. Unsteadiness in the laser or the combustion system confuses temperature measurements considerably, since the measurement often takes several minutes. Longer measurements, taken during high background levels, have high uncertainties. Of course, spontaneous Raman measurements are severely restricted by soot-laden flows where levels of laser-induced fluorescence and laser-modulated particle incandescence are high. This and other temperament difficulties are again related to the weakness of the Raman signal. Measurements have been made, however, in propane (IC) engines and combustion tunnels and clean turbulent diffusion flames. Work initiated by the Nuclear Regulatory Commission 
(NRC) on measurements in super-heated steam (in single and nonequilibrium twophase flows) has been relatively successful as well (Anastasia et al. 1982). Near-Resonant Raman

Near-Resonant excitation could lead to considerable increases in Raman scattering cross sections (likelihoods) and thus in Raman intensities. In this technique, the laser frequency is tuned close to an electronic resonance of the molecule being probed, which can enhance the signal by several orders of magnitude. Thus it raises the signal for one species considerably, which is especially helpful in distinguishing between species with similar vibrational structures (and, hence similar vibrational Raman spectra). The technique is difficult to implement, however. Furthermore, its usefulness is limited since, as noted earlier, most major combustion species only have strong absorption bands below 2000 angstroms, beyond the visible and below the current limitation for tunable lasers. Use of near-resonant techniques for the more accessible minority species is compromised by their low concentrations, limited laser energies, and the need to probe a specific vibrational/rotational state. Diagnostic applications to combustion are not immediately likely.

Ramanography

Most spontaneous Raman work has been limited to single point measurements, or to a few points along a line. Mapping of gas-species concentration in an entire plane has been performed using Raman scattering, however. This technique is called Ramanography. An early effort involved photographing the image-intensified Raman light from a high-pressure gas mixture (Hartley 1974). Improved versions have used a multipass laser approach with a twodimensional multichannel optical analyzer. One recent effort involved measurements in a clean turbulent diffusion flame (Long, Fourguette and Escoda 1983). Although the technique has seen little use because of signal strength constraints, continued improvements in approaches may lead to time-resolved measurements of many nonluminous turbulent fields. 


\subsection{CARS AND OTHER NONLINEAR RAMAN TECHNIQUES}

Coherent anti-Stokes Raman spectroscopy (CARS) is a nonlinear light-mixing process that has rapidly become a prominent method for combustion and gas phase diagnostics. The effect was still in the realm of nonlinear optics research when Taran and others in France pioneered its use for combustion work in the early and mid 1970s. It has since been demonstrated in practical combustors, and industrial applications (temperature measurements) are fast approaching. The primary motivation for the continuing development effort is that CARS can probe the high-interference environments of typical practical combustors. There are several reasons for this (Rahn, Mattern and Farrow 1981). CARS, unlike spontaneous Raman, gives relatively strong signal levels. These signals are coherent beams, which permit high signal collection efficiencies. Interferences are avoided both spatially and because CARS signals are upshifted in frequency from those of the lasers used. This results in ratios of signal to interference that are far higher than for spontaneous Raman. The CARS technique, although difficult and expensive to implement, can probe practical combustors over a wide range of operating conditions, yielding temperature and perhaps major species measurements. Enhanced versions and a different thirdorder nonlinear technique called inverse Raman are also discussed in this section.

\section{Background}

CARS and inverse Raman are based, as are Raman, Rayleigh and fluorescence spectroscopy, on examination of scattered light from the polarization field induced in a molecule by incident light beams. Raman, Rayleigh and fluorescence are first-order (e.g., linear) effects with polarizability linearly dependent on the optical (light) field strength (although fluorescence becomes nonlinear because of quenching). Higher order "susceptibilities," as they are called, exist as well. Such weaker polarizations, however, were not discovered until high-powered pulse lasers were invented in the 1960s. These susceptibilities are material properties, dependent on the phase as well as identity of the material.

Isotropic (uniform) gases have no second-order susceptibility because of their symmetry properties, though interfaces such as solid surfaces do. Gases 
do have third-order polarization susceptibility, in which the polarizability (P) has cubic dependence on the optical (E) field strength (i.e., P is proportional to $E^{3}$, and is of course nonlinear. Despite the basic weakness of higher-order processes, high-powered lasers can be used to stimulate resonances in third-order processes that produce strong signals. It is this signal strength that makes CARS and inverse Raman viable diagnostic processes. The details of CARS will be examined next; inverse Raman, which is a very new process, will be discussed later in the section. There are many other nonlinear processes related to the Raman process, but they are not currently the basis for much practical diagnostics work.

In CARS, the incident lasers at two different frequencies (called the pump beam $\left(W_{1}\right)$ and the stokes beam $\left(W_{2}\right)$ ) interact through the third-order nonlinear susceptibility detailed above. This forced interaction generates a polarization field that produces coherent (beamlike) radiation at a third frequency, $W_{3}$ $=2 W_{1}-W_{2}$. If the frequency difference $\left(W_{1}-W_{2}\right)$ between the incident beams is close to a Raman resonance being probed, the magnitude of the CARS signal is high and $W_{3}$ will be the anti-Stokes frequency relative to the pump beam $W_{1}$. It is thus higher than $W_{1}$ by the difference $W_{1}-W_{2}$. Figure 5.5 shows the basic scheme for CARS measurements.

A CARS spectrum displays the intensity of the CARS beam over a range of frequencies and can be generated in two different ways. A narrow-band Stokes source $W_{2}$ can be scanned through a range, generating the spectrum bit by bit; or a broad-band Stokes laser can be used to generate the entire spectrum in one pulse, which might on occasion permit one-shot measurements of medium properties. From the spectrum, species concentrations can be found (with significant uncertainty) from the intensity of the CARS signal, or, in limited cases (and uniquely to CARS), from the spectrum shape. Temperature measurements, discussed later, also are derived from spectrum shape. Computer simulations of the spectra serve as the references for these measurements. 
Low Resolution CARS Spectrum
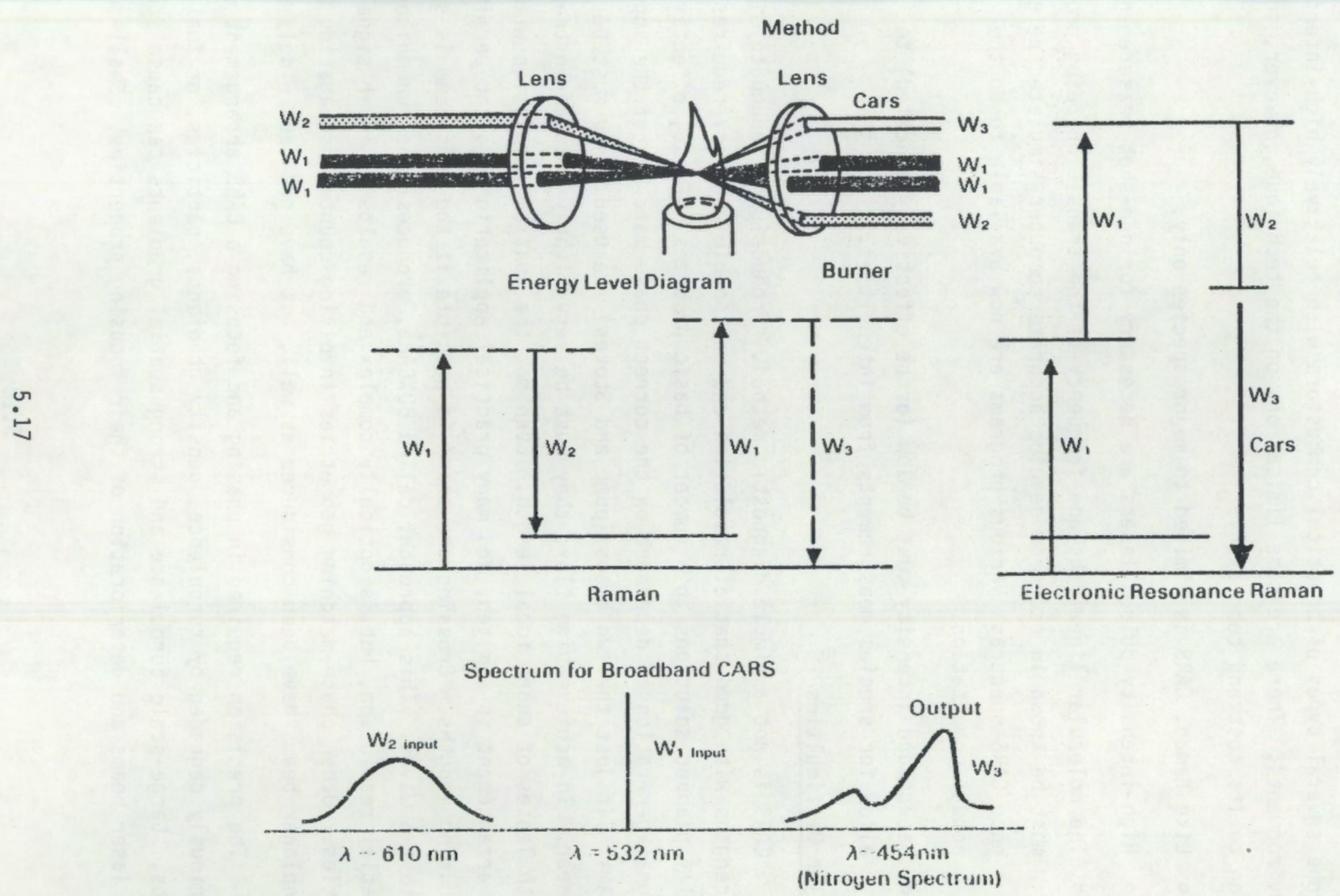

FIGURE 5.5. Basics of CARS Measurement 
Conditions

The signal strength and coherence of CARS have been shown to effectively probe several types of practical combustors with relatively high-interference environments. There are some limitations on the technique, however, in addition to its cost and complexity:

- Like Raman, CARS is limited to major species only.

- High-intensity pulsed lasers are necessary for one-shot measurements.

- The molecular linewidths and frequency of the transition being probed must be known in order to develop accurate computer-simulated reference CARS spectra. Various programs are now available to do the actual computation.

- Background intensity must be low (or be effectively canceled) to allow for species measurements from intensities.

\section{Major Difficulties}

CARS is not a simple diagnostic method. Production of adequate signal strengths with good spatial resolution and reasonable accuracy requires carefully planned solutions to a number of basic problems. First, effective CARS signal generation is dependent on the correct phase matching of the input laser beams. If just the two beams (pump and Stokes) are used, they must be collinear. To achieve high flux, they must be very tightly focused in the sample with lenses of short focal length. Even so, the spatial resolution with such an arrangement is limited, and many practical applications do not permit short focusing lengths. Crossing the beams is possible if the pump beam is split into two waves. This approach, called BOXCARS, produces fine, unambiguous spatial resolution, but is optically complex and results in lower signal intensities. Other phase-matching geometries involving counter-propagating beams or nonplanar beams have been considered as well, but have not been widely used.

The precision required in phasing and focusing a CARS arrangement can be seriously degraded by turbulence, density gradients, particles, or fuel droplets. Large-scale turbulence and strong spatial gradients can cause steering of laser beams and deterioration of their focusing properties. Small-scale 
turbulence can degrade phase front coherence and can reduce signal intensities over long path lengths. Droplets and soots attenuate, scatter and bend laser beams. Particle incandescence can interfere with the CARS signal, especially at high power levels. Such distortions can preclude CARS probing--and any other optical probing as well. The averaging of results that vary widely because of turbulence is also difficult because of the nonlinear variance of the CARS level with both temperature and density.

Another problem with CARS is the presence of frequency-independent (nonresonant) background polarization susceptibility, which gives a nonzero baseline to CARS signals. Weak signals fade into this background, which, if no fix is made, sets the lower limit on CARS species detectability. This "noise" is an important factor, because when such background is significant it must be suppressed by careful orientation of the input polarizations and the output CARS-viewing polarizations. This weakens the signal strength, however, and may not be completely successful in more practical environments. The use of more powerful lasers is limited by a variety of factors: laser tunability and cost, the gas breakdown threshold of the volume being probed, and the intensity limits of the optics being used. Another technique to increase detectability may be developed, however, which involves electronic resonance enhancement of the molecular response. It will be discussed shortly.

Even when the CARS process can be demonstrated (this can sometimes be quite straightforward), making precise and reproducible measurements with it is a difficult task. CARS with pulsed sources suffers from poor reproducibility because of laser power and frequency instabilities. Anti-Stokes power fluctuations of $30 \%$ occur even in well-engineered systems, unless precautions like reference measurements (with the same lightwave set) are taken. These variations severely impact concentration measurements based on signal intensity. Secondly, limited spectral knowledge of the molecules being probed, most particularly knowledge of their exact linewidths, can severely affect concentration measurement accuracy. Attempts to get stronger signals by increasing laser intensity in especially difficult measurement environments are often foiled by gas breakdown or unwanted signal saturation. An additional complication is that at high pressure (such as IC engines), although the signal is 
stronger, the spectrum also changes shape. Finally, there are a few difficulties specific to CARS thermometry that have been reserved for a separate section. As this long list of difficulties makes clear, spontaneous Raman should be used whenever possible because of the complexity of CARS. Figure 5.6 provides an example of the complicated experimental arrangement necessary. The . limitations of spontaneous Raman dictate when CARS must be used. Current Work

CARS species measurement has not progressed as far as thermometry (discussed next), but numerous experiments in simple flows have been completed. Work on spectroscopic studies in such cases as $\mathrm{CH}_{4}, \mathrm{~N}_{2}, \mathrm{O}_{2}$, and $\mathrm{C}_{2} \mathrm{H}_{2}$ has progressed, although much work has been at high temperatures to help with thermometry. Flames and their major constituents such as $\mathrm{CO}$ and $\mathrm{O}_{2}$ have undergone some research, verified by microprobe sampling. Practical measurements have been made as well, in IC engines (Stenhouse et al. 1979), diesels (Kajiyama et a1. 1982), in electric discharges in a gasifier (Taylor 1984) and in a jet engine afterburner (Eckbreth et al. 1983).

Thermometry

CARS temperature measurements have been completed in numerous applications. They are based on the shape of the CARS spectrum of a gas especially selected for thermometry (see Figure 5.7). $N_{2}$ is generally used because it is a major constituent in all air/fuel combustion environments and has been studied fairly thoroughly. Other molecules used include $\mathrm{H}_{2}, \mathrm{CO}, \mathrm{O}_{2}$, and $\mathrm{H}_{2} \mathrm{O}$. Thermometry has specific difficulties related to the general areas discussed earlier. In addition to the normal problems such as particle incandescence and laser focusing and phase matching in complex flows, care must also be taken to provide thermal uniformity in the sampled volume. Turbulence can destroy any chances of uniformity, as well as change the temperature in the volume between pulses. Averaging is thus made difficult because of the spectrum's nonlinear dependence on temperature. Measurements have been made successfully in relatively hostile combustion environments, however, beginning with jet combustors and IC engines in the late 1970s. (Unfortunately, some parts of engine cycles cannot be measured, due to strong gradients and their effects on the probing beams.) Even a diesel engine has been studied through part of its cycle, 


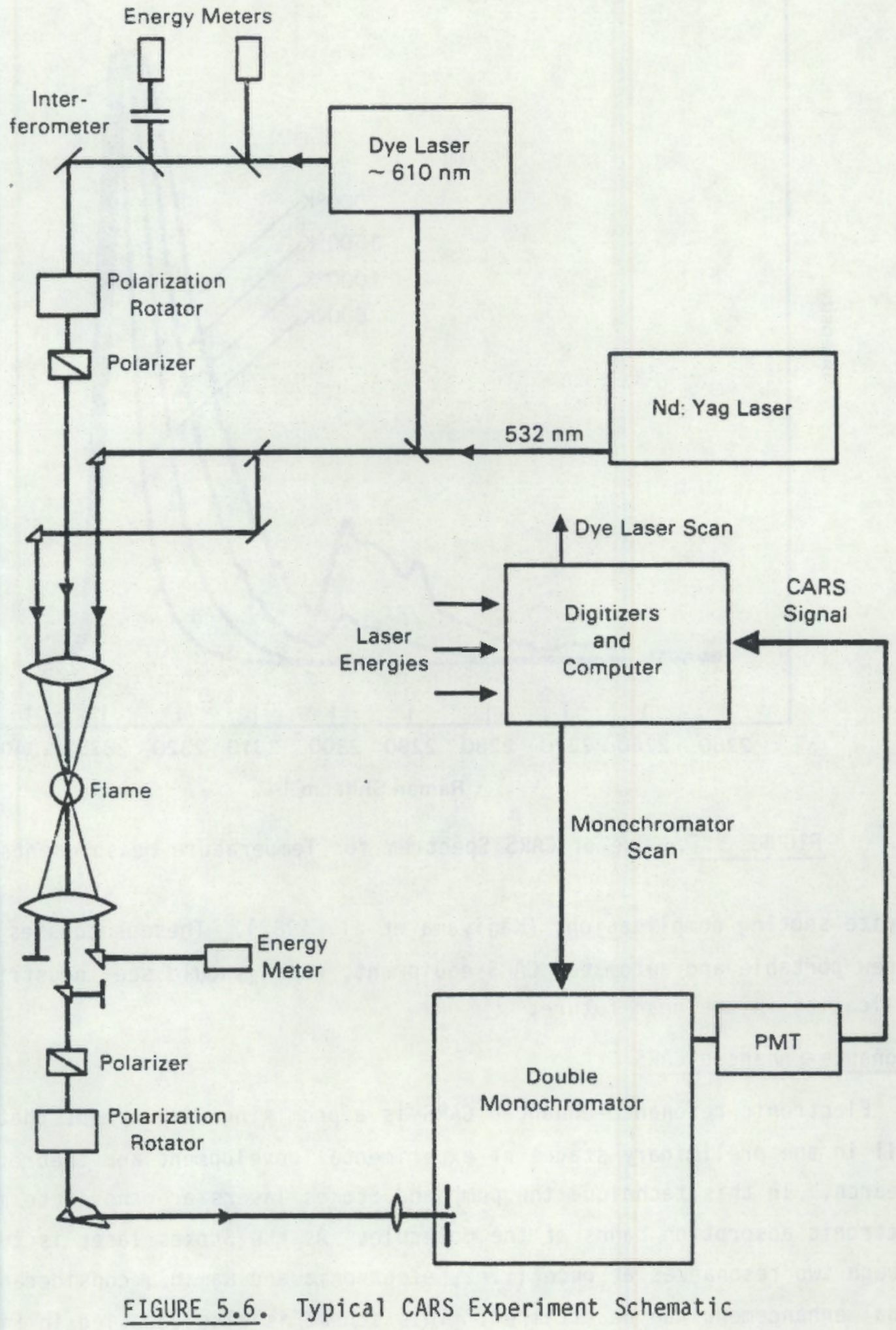




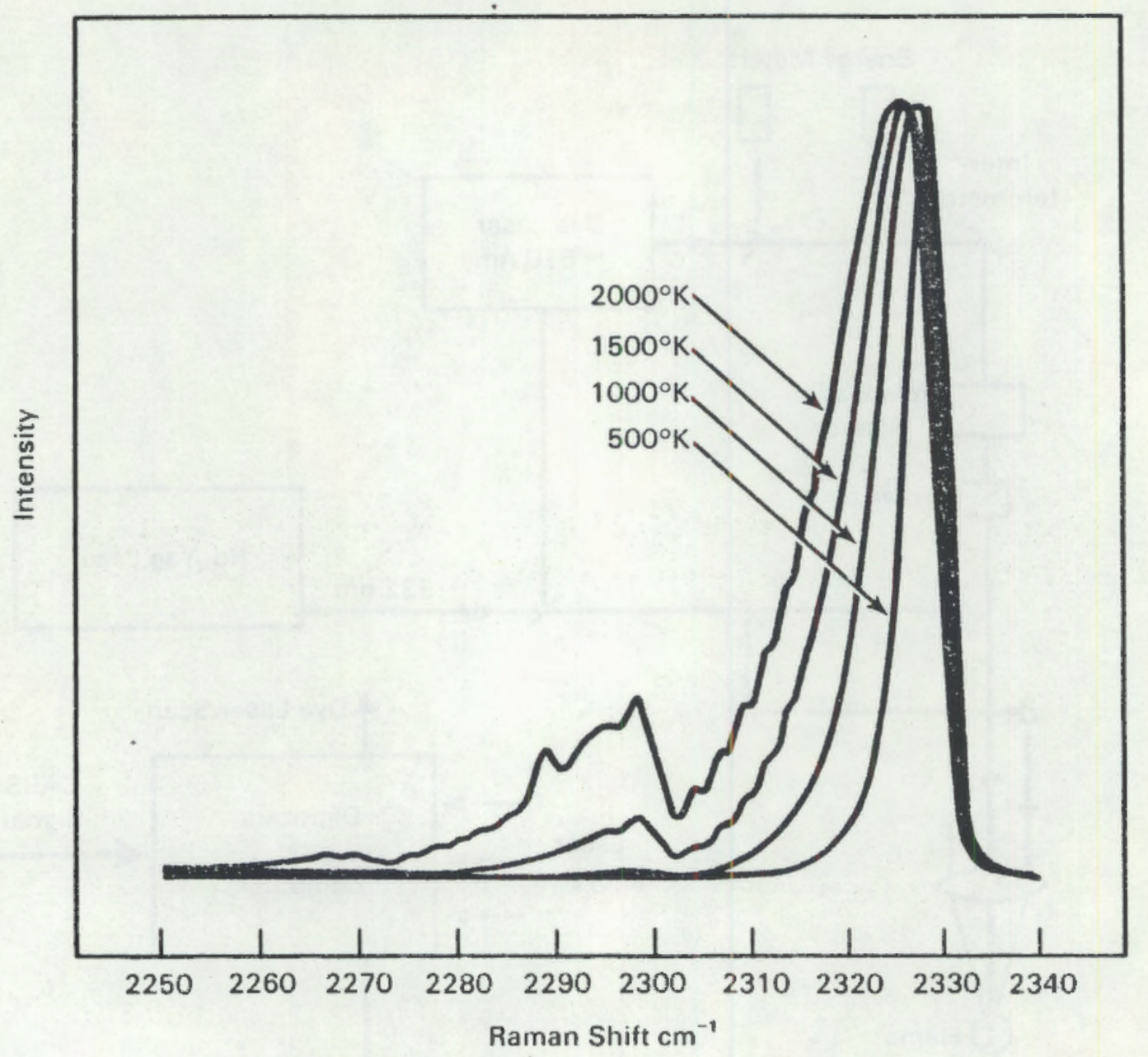

FIGURE 5.7. Use of CARS Spectrum for Temperature Measurements

despite sooting complications (Kajiyama et al. 1982). These successes have led to new portable and automated CARS equipment, which should see industrial application in the near future.

Resonance-Enhanced CARS

Electronic resonance-enhanced CARS is a promising development that is still in the preliminary stages of experimental development and theoretical research. In this technique the pump and Stokes lasers are chosen to match electronic absorption bands of the molecule. As the Stokes laser is tuned through two resonances at once (i.e., electronic and Raman), considerable signal enhancement can be attained. This scheme is also detailed in Figure 5.5. It is, however, an exceedingly difficult experiment, both to perform and 
to interpret. Work on the method is proceeding at the same French laboratory where CARS was originally developed by Taran and others (Attal, Pealat and Taran 1980). Major problems include the imprecision of current dye laser tuning techniques, the instability of the lasers, and insufficient theoretical work on the interactions of the various resonance states. Detectability gains in preliminary measurements are two to three orders of magnitude.

\section{Inverse Raman Spectroscopy}

Inverse Raman Spectroscopy (IRS) is another third-order, stimulated nonlinear process now being explored for its potential as a combustion diagnostic. It has several advantages over CARS. Unlike CARS, in which a signal beam is generated, inverse Raman involves modulation of a probe beam. A pump beam induces absorption in a probe beam at the anti-Stokes frequency via the thirdorder polarization susceptibility. This is also called "stimulated Raman loss spectroscopy." Raman gain spectroscopy, where gain in a probe beam at a Stokes frequency shift from the pump bearn is measured, may also prove useful. Because the methods involve modulation, no phase matching is required. Spectral interpretation is simpler than CARS because the spontaneous Raman spectrum is being recorded. Especially at high pressure, the gain/loss can be quite large, permitting broadband probes and one-shot detection. For flame probing, the technique is slightly more sensitive than CARS, because of its spectral character and resolution. IRS has been used for gathering fundamental spectroscopic data in flames $\left(\mathrm{N}_{2}, \mathrm{CO}, \mathrm{H}_{2}\right)$ as well as line-width data (Rahn 1982). This information is vital for CARS and other Raman measurements. Thus, IRS may fill a growing role in combustion research.

\subsection{OTHER SPECTROSCOPIES}

This section briefly discusses several more spectroscopic methods at varying degrees of development. They were not closely enough related to the major methods covered so far to be included in earlier sections. Neither are they closely related to each other. They do have enough potential to be worth including in this survey, however, and are therefore discussed here. The first 
method is Rayleigh (elastic) scattering, which is already familiar as a simplification of the Mie theory applied to particles. It is also a useful diagnostic phenomenon for gases. Next are several sensitive methods useful in similar applications to LIF. One of these is absorption, an old standby line-of-sight method. Optogalvanic and photoacoustic spectroscopy excite molecules with lasers (as does LIF), but then detect the resultant electric field or the sound rather than the fluorescence. Photothermal spectroscopy is closely related to photoacoustic spectroscopy. Instead of detecting a pressure wave some distance from its source, this method detects the density difference that causes the sound. The newer methods (the final three listed here) are being developed as possible alternatives to LIF. Another new method takes advantage of an occurrence that most methods must avoid, i.e., laser-induced breakdown of the medium under study (and the spectra of the sparks this produces), to identify complex molecules.

There is always a search for increased sensitivity and accuracy in the field of diagnostics development. These are generally gained at considerable cost, nowever, so judgments must be made on how far to explore each avenue. These judgments have yet to be made on the new methods listed here, just as on inverse Raman, resonance CARS, and others.

Rayleigh

This section discusses the application of Rayleigh theory to gases; its use for small particles was outlined in Chapter 4. Rayleigh scattering is elastic--at the wavelength of the incident light--so the Rayleigh line is the combined scattering from all the species in the sample. Its intensity depends on the scattering properties and fraction of each species present. These properties (the scattering cross sections) can be readily calculated, and they are much higher than Raman cross sections, so Rayleigh has wide application possibilities. It is used in combustion for measurements of number density and temperature. The scattering power depends on number density, which can be related to temperature by an equation of state. The measurement may often only be semiquantitative, however, since scattering cross sections depend on composition, which may vary (e.g., in a flame). Experimental conditions are often 
manipulated to mitigate this concern. Another difficulty is interference by light scattering from dust or soot.

Rayleigh measurements have seen considerable recent use in turbulent flames, especially to provide data for turbulence closure models (Dibble and Hollenbach 1981) and for details of flame instability. This popularity has resulted from the method's high data rates, which preclude use of slower techniques popular in laminar flames. Further practical applications are likely, particularly for temperature profiles--perhaps even in two dimensions, using arrays of detectors.

Absorption

Absorption is a simple technique, both experimentally and theoretically, which involves measuring variations in the level of light transmitted through the medium. Its data (on species concentration or temperature) are therefore integrated over the line of sight of the instrument. The measurement is based on the relationship between the absorption coefficient (at a given frequency) and the electron populations in the transition corresponding to that frequency. The intensities of these transitions give temperature information. The frequency used is chosen to be specific to the substance whose concentration is desired. This procedure requires only relatively simple quantum mechanical calculations, similar to those for emission spectra.

The major complication of the absorption technique is the effect of lineshape (the intensity distribution over frequency of the laser and changes from collisions and Doppler shifts), which requires accurate analytical algorithms. Such algorithms are now available, however.

It must be noted that absorption measurements predate lasers, and conventional light is cheaper and has a wider spectral range, but lasers give much better resolution and data rates. Typical measurements possible with lasers involve temperature or radical concentrations in flames, including various hydrocarbons, as well as $\mathrm{OH}$, NO and $\mathrm{CO}$. There are some novel variations of absorption (e.g., saturation) to provide superior sensitivity, but they are generally unstable or difficult to interpret and are not discussed here. In 
addition, tomography has been used to create spatially precise concentration mappings from a sequence of absorption measurements.

\section{Ionization (Optogalvanic) Spectroscopy}

"Optogalvanic" is a term signifying changes in the electrical properties of plasma due to photo-excitation. In this technique a species is laser excited to a resonant state close to its ionization limit, and then goes through a collisional or photo ionization. This produces a change in the plasma conductivity that can be sensitively measured. Past efforts have focused on detecting metals doped into flames. The method does lack spatial resolution compared with LIF, but can make the same type of measurements with comparable sensitivity. It could be an alternative to LIF when high fluorescence levels block LIF measurements. Like LIF, it can be used with multiphoton excitation.

Photoacoustic and Phototherma1 Spectroscopy.

These techniques are based on detecting the energy that a sample absorbs from the laser; they are in a sense the detailed complement to absorption. With powerful lasers, very good signals derive from very small absorptions. The photoacoustic method detects the pressure wave (sound) that develops when absorbed energy converts to translational energy. Excellent detectability is possible--even absorptions by soot have been measured. It is of limited usefulness in flames, however; despite its sensitivity, it is not spatially resolved, and signals must pass through a hot, nonuniform path to reach the microphone.

Photothermal spectroscopy improves on photoacoustic by detecting the density change close to the pump beam providing the absorbed energy. A second laser is used, crossing the first, which is deflected by the thermal wave produced by the pump beam, as shown in Figure 5.8. Extremely small deflections can be sensed to produce concentration or temperature measurements. This technique would be difficult, however, in turbulent flows that could perturb the probed beam by themselves. 


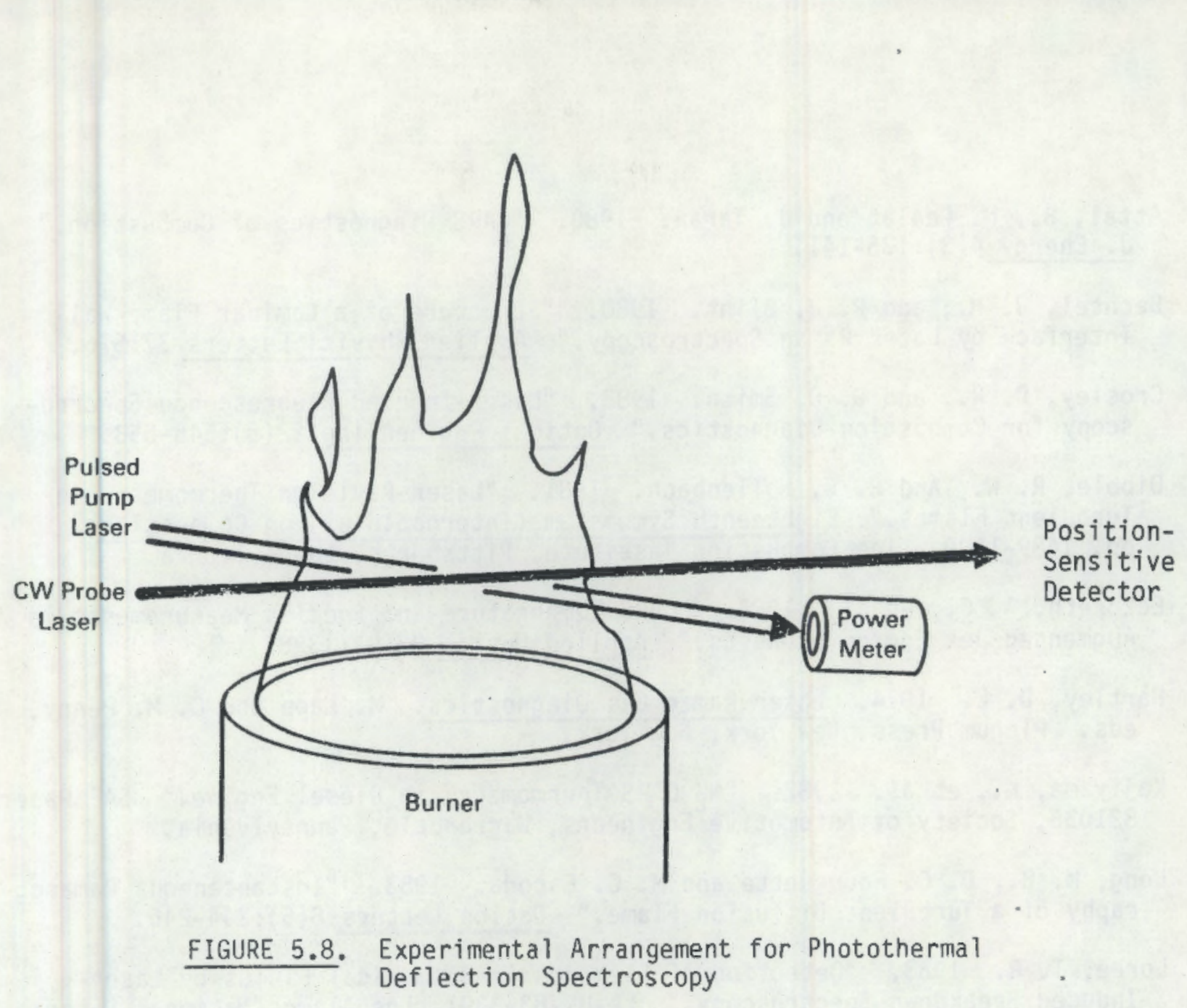

Laser-Induced Breakdown Spectroscopy

High-powered lasers can generate "sparks" (a small plasma) due to gas breakdown, a fact which imposes limitations on most conventional diagnostics. This breakdown process has been the subject of considerable nonlinear optics research since its first observation. The emission spectra from these sparks are atomic and ionic lines, which, with care, can be analyzed. Elemental analyses have been performed for hydrogen, carbon, nitrogen, and oxygen, and also for methane-air systems. Various applications to complex molecules including blood constituents and various pollutants are being developed at Los Alamos National Laboratory (Loree 1983).

\subsection{REFERENCES}

Anastasia, C. M., et al. 1982. "Raman Scattering Measurements for Water Vapor in Nonequilibrium Dispersed 2-Phase Flow." NUREG/CR-2905, U.S. Nuclear Regulatory Commission, Washington, D.C. 
Atta1, B., M. Pealat and J. Taran. 1980. "CARS Diagnostics of Combustion." J. Energy 4(3):135-141.

Bechtel, J. H., and R. J. Blint. 1980. "Structure of a Laminar Flame-Wall Interface by Laser Raman Spectroscopy." Applied Physics Letters 37:576.

Crosley, D. R., and G. D. Smith. 1983. "Laser-Induced Fluorescence Spectroscopy for Combustion Diagnostics." Optical Engineering 22(5):545-553.

Dibble, R. W., and R. E. Hollenbach. 1981. "Laser Rayleigh Thermometry in Turbulent Flames." Eighteenth Symposium (International) on Combustion, pp. 1489-1499. The Combustion Institute, Pittsburgh, Pennsylvania.

Eckbreth, A. C., et al. 1984. "CARS Temperature and Species Measurements in Augmented Jet Engine Exhausts." Applied Optics 23(9):1328.

Hartley, D. L. 1974. Laser Raman Gas Diagnostics. M. Lapp and C. M. Penny, eds. Plenum Press, New York, New York.

Kajiyama, K., et al. 1982. "N $\mathrm{N}_{2}$ CARS Thermometry in Diesel Engine." SAE Paper 821036, Society of Automotive Engineers, Warrendale, Pennsylvania.

Long, M. B., D. C. Fourguette and M. C. Escoda. 1983. "Instantaneous Ramanography of a Turbulent Diffusion Flame." Optics Letters 8(5):244-246.

Loree, T. R. 1983. "Detection of Elements in Biomedical Fluids by LaserInduced Breakdown Spectroscopy." LA-UR-83-3391, Los Alamos National Laboratory, Los Alamos, New Mexico.

McDaniel, J. C., B. Hiller and R. K. Hansen. 1983. "Simultaneous MultiplePoint Velocity Measurements Using Laser-Induced Fluorescence." Optics Letters 8:51-53.

Rahn, L. A., P. L. Matten and R. L. Farrow. 1981. "A Comparison of Coherent and Spontaneous Raman Combustion Diagnostics." Eighteenth Symposium (International) on Combustion, pp. 1533-1542. The Combustion Institute, Pittsburgh, Pennsylvania.

Rahn, L. A. 1982. "Time-Resolved Inverse Raman Spectroscopy." Optics Letters $7(2): 66-68$.

Stenhouse, I. A., et al. 1979. "CARS Measurements in an Internal Combustion Engine." Applied Optics 18(22):3819.

Taylor, D. J. 1984. "CARS Concentration and Temperature Measurements in Coal Gasifiers." LA-UR-83-1840, Los Alamos National Laboratory, Los Alamos, New Mexico. 


\subsection{BIBLIOGRAPHY}

\section{Fluorescence:}

Alden, M., et al. 1983. "Spatially Resolved Temperature Measurements in a Flame Using Laser-Exited Two-Line Atomic Fiuorescence and Diode-Array Detection." Optics Letters 8(5):241-243.

Bechtel, J. H. 1979. "Temperature Measurements of the Hydroxyl Radical and Molecular Nitrogen in Premixed Laminar Flames by Laser Techniques." Applied Opti:s 18:2100.

Bischel, H. K., B. E. Perry and D. R. Crosley. 1981. "Two-Photon LaserIndused Fluorescence in Oxygen and Nitrogen Atoms." Chemical Physics Letters $82(1): 85-88$.

Bischel, W. K., B. E. Perry and D. R. Crosley. 1982. "Detection of Fluorescence from 0 and $\mathrm{N}$ Atoms Induced by Two-Photon Absorption." Applied Uptics $21: 1419-1429$.

Cattolica, R. J. 1981. "OH Rotational Temperature from Two-Line Laser-Excited Fluorescence. Applied Optics 20:1156.

Crosley, D. R. 1981. "Collisional Effects on Laser-Induced Fluorescence Flame Measirements." Optical Engineering 20(4):511-521.

Crosley, D. R. 1982, "Laser-Induced Fluorescence in Spectroscopy, Dynamics, and Diagnostics." Journal of Chemical Education 59:446.

Dyer, M. J., and D. R. Crosley. 1982. "Two-Dimensional Imaging of OH LaserInduced Fluorescence in a Flame." Optics Letters 7(8):382-384.

Kowalik, R. M., and C. H. Kruger. 1979. "Laser Fluorescence Temperature Measurements." Combustion and Flame 34:135-140.

Kychakoff, 0., R. D. Howe and R. K. Hanson. 1984. "Quantitative Flow Visualization Technique for Measurements in Combustion Gases." Applied Optics $23(5): 704-712$.

Lucht, R. P., et al. 1984. "Single-Pulse, Laser-Saturated Fluorescence Measurements of $\mathrm{OH}$ in Turbulent Non-Premixed Flames." Optics Letters $9(3): 90-02$.

Lucht, R. P., D. H. Sweeney and N. M. Laurendeau. 1983. "Laser Saturated Fluorescence Measurements of $\mathrm{OH}$ Concentration in Flames." Combustion and Flame 50:189.

Schofield, K., and M. Steinberg. 1981. "Quantitative Atomic and Molecular Laser Fluorescence in the Study of Detailed Combustion Processes." Optical Engineering 20:501. 
Raman:

Birkbeck, J. C. 1982. "Raman Spectroscopy." MCM-2896, Monsanto Mound Facility, Miamisburg, Ohio.

Boiarski, A. A., R. H. Barnes and J. F. Kircher. 1978. "Flame Measurements Utilizing Raman Scattering." Combustion and Flame 32:111.

Drake, M. C., R. W. Bilger and S. H. Starner. 1982. "Raman Measurements and Conserved Scalar Modeling in Turbulent Diffusion Flames." Nineteenth Symposium (International) on Combustion. The Combustion Institute, Pittsburgh, Pennsylvania.

Johnston, S. C. 1979. "Precombustion Fuel/Air Distribution in a Stratified Charge Engine Using Laser Raman Spectroscopy." SAE Paper 790433, Society of Automotive Engineers, Warrendale, Pennsylvania.

Lapp, M., and D. L. Hartley. 1976. "Raman Scattering Studies of Combustion." Combustion Science and Technology 13:199.

Lapp, M. and C. M. Penney. 1974. Laser Raman Gas Diagnostics. Plenum Press, New York, New York.

Smith, J. R. 1980. "Temperature and Density Measurements in an Engine by Pulsed Raman Spectroscopy." SAE Paper 800137, Society of Automotive Engineers, Warrendale, Pennsylvania.

Smith, J. R. 1982. "Turbulent Flame Structure in a Homogeneous Charge Engine." SAE Paper 820043, Society of Automotive Engineers, Warrendale, Pennsylvania.

\section{CARS and Nonlinear Raman Techniques}

Allessandretti, G. C., and P. Violino. 1983. "Thermometry by CARS in an Automobile Engine." J. Phys. D. Applied Phys. 16:1583-1594.

Eckbreth, A. C. 1980. CARS Thermometry in Practical Combustors." Combustion and Flame 39:133-147.

Eckbreth, A. C., and R. J. Hal1. 1981. "CARS Concentration Sensitivity With and Without Non-Resonant Background Suppression." Combustion Science and Technology 25:175.

Eesley, G. L. 1979. "Coherent Raman Spectroscopy." J. of Quantitative Spectroscopy and Radiative Transfer 22:507.

Farrow, R. L., P. L. Mattern, and L. A. Rahn. 1982. "Comparison Between CARS and Corrected Thermocouple Temperature Measurements in a Diffusion Flame." Applied Optics 21(17):3119. 
Ferrario, A., M. Garbi and C. Malvicini. 1983. "Rea1 Time CARS Spectroscopy in a Semi-Industrial Furnace." Paper WD2 CLE0, 1983 Meeting. Baltimore, Maryland.

Greenhalgh, D. A. 1983. "RECLAS: Resonant-Enhanced CARS from $C_{2}$ Produced by Laser Ablation of Soot Particles." Applied Optics 22:1128-1130.

Greenhalgh, D. A., F. M. Porter and H. A. England. 1983. "The Application of Coherent Anti-Stokes Raman Scattering to Turbulent Combustion Thermometry." Combustion and Flame 49:171-181.

Hall, R. J. 1979. "CARS Spectra of Combustion Gases." Combustion and Flame $35: 47-60$.

Hal1, R. J. 1983. "CARS Spectroscopic Modeling for Combustion Diagnostics." Optical Engineering 22:322.

Hall, R. J., and A. C. Eckbreth. 1984. "Coherent Anti-Stokes Raman Spectroscopy (CARS): Application to Combustion Diagnostics." In Laser Applications. Academic Press, New York, New York.

Klick, D., K. A. Marko and L. Rimai, 1981. "Temperature and Concentration Measurements by CARS in a Firing Single Cylinder Engine." SAE Paper 810227, Society of Automotive Engineers, Warrendale, Pennsyivania.

Murphree, D. L., et al. 1982. "Optical Diagnostic Techniques for Coal Fired MHD Applications." AIAA Paper 82-0377.

Shen, Y. R. 1976. "Recent Advances in Nonlinear Optics." Reviews of Modern Physics $48(1): 1-32$.

Snow, J. B., J. Zheng and R. C. Chang. 1983. "Spatially and Spectrally Resolved Multipoint Coherent Anti-Stokes Raman Scattering from $\mathrm{N}_{2}$ and $\mathrm{O}_{2}$ Flows." Optics Letters 8(12):599-601.

Verdieck., J. F., R. J. Hall and A. C. Eckbreth. 1983. Electronically Resonant CARS Detection of OH. AIAA Paper 83-1477, American Institute of Aeronautics and Asitronautics, New York, New York.

\section{Other Spectroscopics:}

Cheng, R. K., R. G. Bill and F. Robben. 1981. "Experimental Study of Combustion in a Turbulent Boundary Layer." Eighteenth Symposium (International) on Combustion, pp. 1021-1029. The Combustion Institute, Pittsburgh, Pennsyivania.

Escoda, M. C., and M. B. Long. 1983. "Rayleigh Scattering Measurements of the Gas Concentration Field in Turbulent Sets." AIAA Journal 21(1):81-84. 
Mueller-Dethlefs, K., and F. J. Weinberg. 1978. "Burning Velocity Measurements Based on Laser Rayleigh Scattering." Seventeenth Symposium (Internationa1) on Combustion. The Combustion Institute, Pittsburgh, Pennsylvania.

Namazian, M., et al. 1982. "Two Point Rayleigh Scattering Measurements in a V-Shaped Turbulent Flame." Nineteenth Symposium (International) on Combustion. The Combustion Institute, Pittsburgh, Pennsylvania.

\section{Absorption:}

Cattolica, R. J., S. Yoon and E. L. Knuth. 1982. "On Concentration in an Atmospheric Pressure Methane-Air Flame from Molecular Beam Mass Spectroscopy and Laser Absorption Spectroscopy." Combustion Science and Technology $28: 225$.

Hanson, R. K. 1980. "Absorption Spectroscopy in Sooting Flames Using a Turable Diode Laser." Applied Optics 16:1479.

Kychakoff, G., et al. 1982. "Spatially Resolved Combustion Using Crossed-Beam Saturated Absorption Spectroscopy." Conference on Lasers and ElectroOptics. (AES 1982).

Wang, J. Y. 1976. "Laser Absorption Methods for Simultaneous Determination of Temperature and Species Concentration Through a Cross Section of a Radiating Flow." Applied Optics 15:768.

\section{Optogalvanic:}

Goldsmith, J. E. M., and J. E. Lawler. 1981. "Optogalvanic Spectroscopy." Contemporary Physics 22:255.

Goldsmith, J.E. M. 1982. "Resonant Multiphoton Optogalvanic Detection of Hydrogen in Flames." Optics Letters 7:437.

Mallard, W. G., J. H. Miller and K. C. Smyth. 1982. "Resonantly Enhanced TwoPhoton Photo-Ionization of NO in an Atmospheric Flame. J. Chemical Physics $76: 3403$.

\section{Photoacoustic and Photothermal:}

Allen, J. E., Jr., W. R. Anderson and D. R. Crosley. 1977. "Optoacoustic Pulses in a Flame." Optics Letters 1(4):118.

Jackson, W. B., et al. 1981. "Photothermal Detection Spectroscopy and Vetection." Applied 0ptics 20:1344.

Patel, C. K. N., and A. C. Tam. 1981. "Pulsed Optoacoustic Spectroscopy of Condensed Matter." Reviews of Modern Physics 53:517. 
Roessler, D. M. 1982. "Opacity and Photoacoustic Measurements of Diese1 Particle Mass Emissions." SAE Paper 820460, Society of Automotive Engineers, Warrendale, Pennsylvania.

Rose, A., et al. 1982. "Applications of Photothermal Defection Technique to Combustion Diagnostics." Applied Optics 21:2663.

Tennal, K., G. J. Salamo and R. Gupta. 1982. "Minority Species Concentration Measurements in Flames by the Photoacoustic Technique." Applied Optics $21: 2133$.

Zapka, W., P. Pokrowsky and A. C. Tam. 1982. "Noncontact Optoacoustic Monitoring of Flame Temperature Profiles." Optics Letters 7:477. 
No. of

Copies

OFFSITE

25

M. Gunn

U.S. Department of Energy

Energy Conversion and

Utilization Division

1G-080 Forrestal Building

Washington, DC 20585

J. Brogan

U.S. Department of Energy

Office of Energy Systems Research

Washington, DC 20585

27 DOE Technical Information Center

R. Abarcar

Energetics, Inc.

9210 Route 108

Columbia, MD 21045

M. Allen

HTGL

Building 520

Stanford University

Stanford, CA 94305

R. Barnes

Battelle Columbus Laboratories

505 King Avenue

Columbus, OH 43201

M. Bernard

Argonne National Laboratory

Building 362-2B

Argonne, Illinois 60439

A. Boiarski

Battelle Columbus Laboratories

$505 \mathrm{King}$ Avenue

Columbus, $\mathrm{OH} 43201$
No. of

Copies

T. T. Bramlette

Sandia National Laboratories

P.0. Box 969

Livermore, CA 94550

J. A. Carpenter, Jr.

Oak Ridge National Laboratory

P.O. Box $x$

Building 4508 North Room 263

Oak Ridge, TN 37830

D. Crosley

SRI Internationa?

333 Ravenswood Avenue

Menio Park, CA 94025

T. M. Dyer

Sandia Laboratories

P.0. Box 969

Livermore, CA 94550

J. J. Eberhardt

CE -142

U.S. Department of Energy

1000 Independence Avenue

Washington, DC 20585

Dr. D. R. Hardesty

Sandia National Laboratories Division 8361

Livermore, CA 94550

Prof. P. 0. Hedman

Brigham Young University

$350 \mathrm{CB}$

Provo, UT 84602

S. Hsu

National Bureau of Standards Bidg. 220 Room A-215

Washington, DC 20234 
No. of

Copies

M. Kaminsky

Argonne National Laboratory

9700 South Cass Avenue

Argonne, IL 60439

Dr. M. Lapp

Sandia National Laboratories

Division 8354

Livermore, CA 94550

T. Levinson

CE-142

U.S. Department of Energy

1000 Independence Avenue

Washington, DC 20585

C. W. Robinson

Sandia Laboratories

P.0. Box 969

Livermore, CA 94550

R. Sawyer

Mechanical Engineering Department

University of California

Berkeley, CA 94720
No. of

Copies

Dr. J. C. F. Wang

Sandia National Laboratories

Division 8361

Livermore, CA 94550

ONSITE

Richland Uperations Office

H. E. Ransoin/D. R. Snegna

65 Pacific Northwest Laboratory

W. B. Ashton

H. J. Bomelburg

D. L. Brenchley

M. T. Thomas

W. C. Cliff

D. B. DeBellis

J. M. Bates

R. A. Hutchinson (50)

S. E. Liebetrau

Publishing Coordination $\mathrm{MH}$ (2)

Technical Information Files (5) 